\title{
Hepatic Sirt1 deficiency in mice impairs mTorc2/Akt signaling and results in hyperglycemia, oxidative damage, and insulin resistance
}

\author{
Rui-Hong Wang, ${ }^{1}$ Hyun-Seok Kim, ${ }^{1}$ Cuiying Xiao, ${ }^{1}$ Xiaoling Xu, ${ }^{1}$ \\ Oksana Gavrilova, ${ }^{2}$ and Chu-Xia Deng ${ }^{1}$
}

${ }^{1}$ Genetics of Development and Disease Branch and ${ }^{2}$ Mouse Metabolism Core Laboratory, National Institute of Diabetes and Digestive and Kidney Diseases, NIH, Bethesda, Maryland, USA.

\begin{abstract}
Insulin resistance is a major risk factor for type 2 diabetes mellitus. The protein encoded by the sirtuin 1 (Sirt1) gene, which is a mouse homolog of yeast Sir2, is implicated in the regulation of glucose metabolism and insulin sensitivity; however, the underlying mechanism remains elusive. Here, using mice with a liver-specific null mutation of Sirt1, we have identified a signaling pathway involving Sirt1, Rictor (a component of mTOR complex 2 [mTorc2]), Akt, and Foxo1 that regulates gluconeogenesis. We found that Sirt1 positively regulates transcription of the gene encoding Rictor, triggering a cascade of phosphorylation of Akt at S473 and Foxo1 at S253 and resulting in decreased transcription of the gluconeogenic genes glucose-6-phosphatase (G6pase) and phosphoenolpyruvate carboxykinase (Pepck). Liver-specific Sirt1 deficiency caused hepatic glucose overproduction, chronic hyperglycemia, and increased ROS production. This oxidative stress disrupted mTorc2 and impaired mTorc2/Akt signaling in other insulin-sensitive organs, leading to insulin resistance that could be largely reversed with antioxidant treatment. These data delineate a pathway through which Sirt1 maintains insulin sensitivity and suggest that treatment with antioxidants might provide protection against progressive insulin resistance in older human populations.
\end{abstract}

\section{Introduction}

The 7 members of the mammalian sirtuin family (SIRT1-SIRT7) have $\mathrm{NAD}^{+}$-dependent deacetylase activity and/or mono-ADP ribosylase activity (1-4). Their substrates include histones and nonhistone proteins that are involved in numerous biological functions, including cell growth, apoptosis, senescence, neuronal protection, adaptation to calorie restriction, organ metabolism and diseases, DNA damage response and repair, and tumorigenesis (1-3, 5-11).

Functions of sirtuin 1 (SIRT1), a founding member of the sirtuin family, in glucose metabolism have been extensively studied at the whole-organism level. SIRT1 expression is induced upon nutritional stress $(12,13)$. Activation of SIRT1 protein by fasting induces gluconeogenic genes and hepatic glucose production, whereas acute knockdown of SIRT1 in the mouse liver with the adenovirus system reduces glucose output $(14,15)$. It was also reported that SIRT1 knockdown in the liver by using an antisense oligonucleotide decreases basal gluconeogenesis and increases hepatic insulin responsiveness in diabetic rats (16). In contrast, transgenic mice with moderate overexpression of SIRT1 displayed improved glucose tolerance due to decreased hepatic glucose production, suggesting a negative role of SIRT1 in gluconeogenesis (17). Moreover, a recent report using mice carrying a liver-specific deletion of exon 4 of the Sirt 1 gene mediated by albumin-Cre (Sirt1 ${ }^{\text {exon4/exon } 4}$; Alb-Cre mice) revealed normal levels of fasting and fed blood glucose (18). These observations may reflect a complex role of SIRT1 in the regulation of glucose metabolism at different

Conflict of interest: The authors have declared that no conflict of interest exists. Citation for this article: J Clin Invest. 2011;121(11):4477-4490. doi:10.1172/JCI46243. experimental conditions. Consistent with this view, it was shown that SIRT1 represses the high glucose production mediated by P300/CBP through deacetylating CREB-regulated transcription coactivator 2 (CRTC2) upon a short-term fasting (6 hours), whereas after long-term fasting (18 hours), SIRT1 activates gluconeogenesis through deacetylating FOXO1 (19). Thus, activation of SIRT1 can either inhibit or enhance hepatic glucose production through interacting with different proteins at distinct stages during fasting. Nonetheless, the role of SIRT1 in gluconeogenesis under physiological conditions remains controversial and needs further investigation.

Similar inconsistent observations were also reported for liver steatosis associated with SIRT1 loss. Work performed by 2 different groups

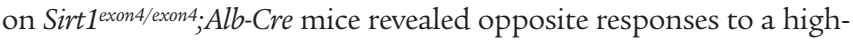
fat diet, in terms of whether SIRT1 loss accelerates (20) or protects from (21) fatty liver formation. While the cause for this discrepancy is unclear, we recently found that Cre-loxP-mediated liver-specific deletion of exons 5 and 6 of the Sirt1 gene (Sirt1 ${ }^{\text {exons-6/exon5-6; }}$ Alb-Cre) resulted in spontaneous liver steatosis that is accompanied by increased expression of carbohydrate-responsive element-binding protein and a number of its downstream genes under a normal feeding condition

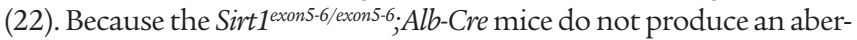
rant shorter form of SIRT1 protein, which is readily detectable in the Sirt1 1exon4/exon4; Alb-Cre mice, it may account for, at least in part, the different phenotypes displayed in these different mutant mice.

Insulin/AKT/FOXO1 represents another important signaling pathway that controls hepatic glucose production and metabolism. In the liver, feeding increases insulin secretion, activating the AKT pathway in hepatocytes, which phosphorylates FOXO1 at S253 


\section{A}

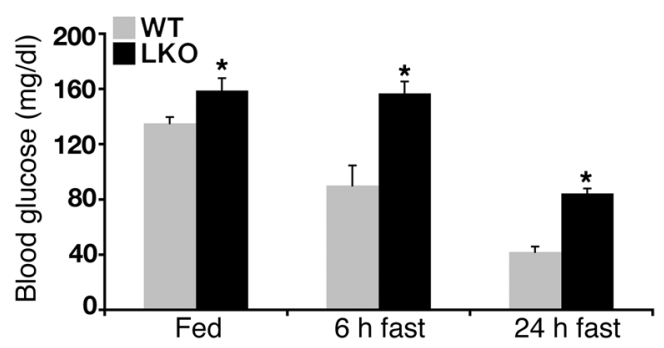

B 250

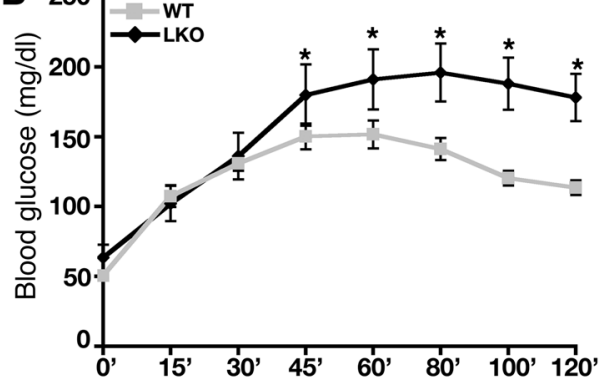

C
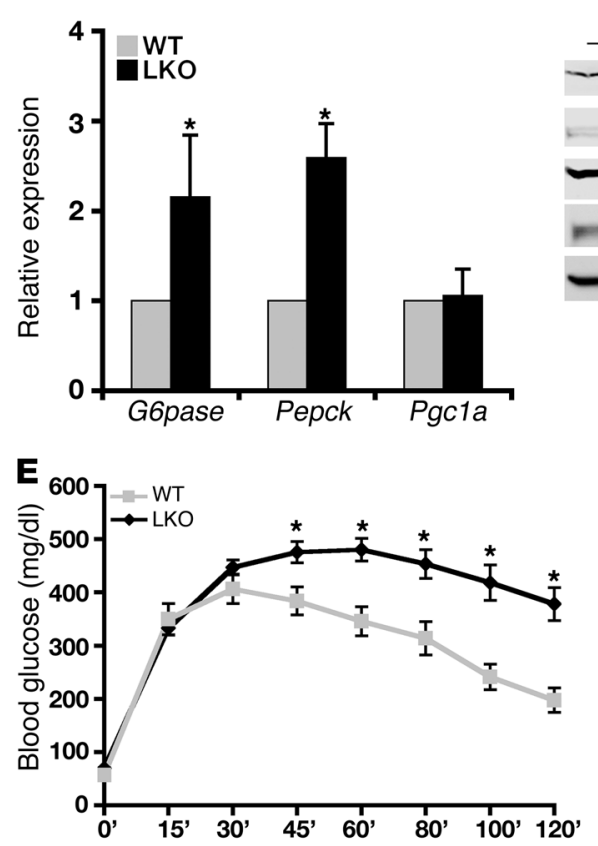

D

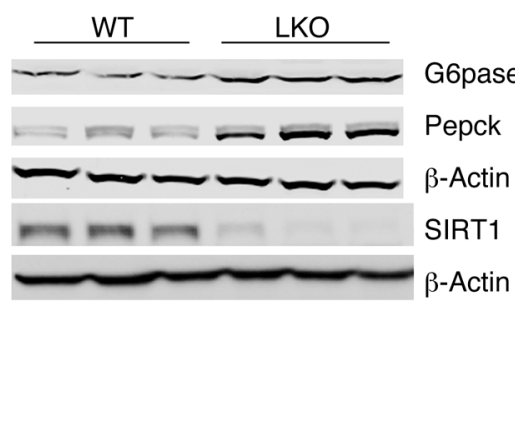

$\mathbf{F}$
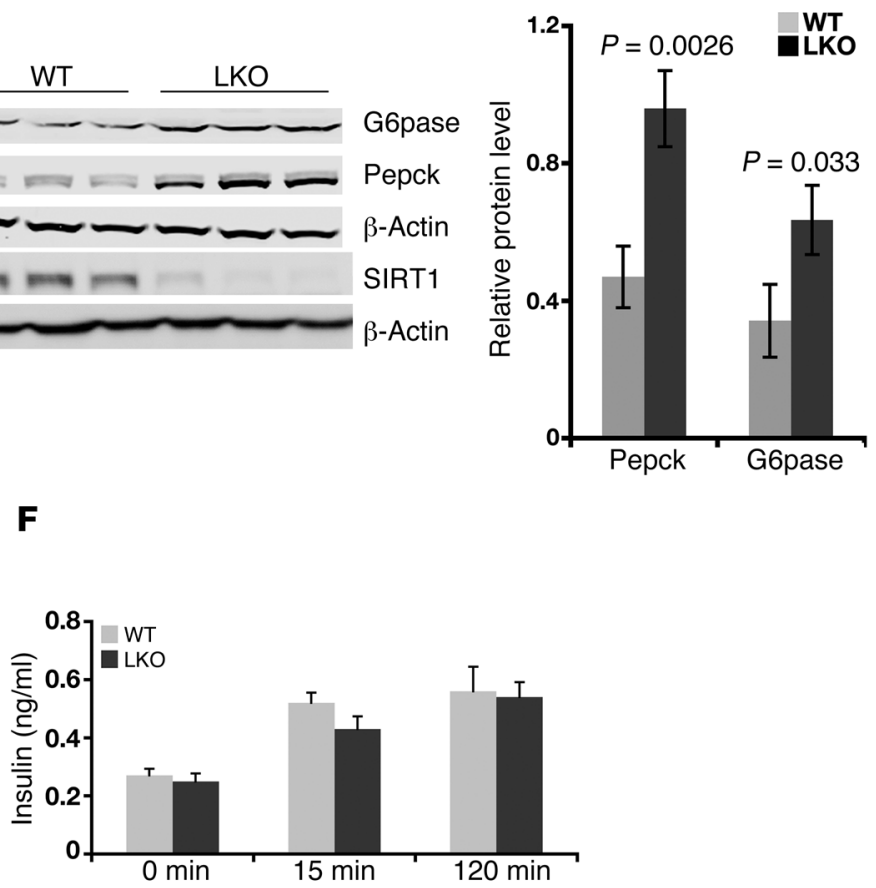

Figure 1

Liver-specific deletion of SIRT1 causes increased hepatic glucose production. (A) Sirt1 ${ }^{L K O}$ animals contain higher blood glucose under fed, 6-hour, and 24-hour fasting conditions than wild-type mice ( $n=39$ pairs of males) measured at 2 months of age. ${ }^{*} P<0.01$, Student $t$ test. (B) PTT shows Sirt ${ }^{\text {LKO }}$ mice $(n=12)$ produce more glucose than wild-type mice $(n=11)$ at 2 month of age. ${ }^{*} P<0.05$. (C) The expression of gluconeogenesis genes (G6pase and Pepck) is increased at the mRNA level $(n \geq 6)$. ${ }^{*} P<0.02$. (D) G6pase and Pepck protein levels are also elevated. Twenty pairs of mice were used. The bar graph on right is the quantification of Western blots from all the samples. (E and F) Sirt1 ${ }^{L K O}$ animals display glucose intolerance. (E) GTT assay was performed in 2-month-old males, and (F) the corresponding blood insulin level during GTT time course was determined ( $n=15$ pairs). ${ }^{*} P<0.01$.

(S253 in mouse and S256 in human) (23). The phosphorylated forkhead box O1 (pFOXO1) is then relocated to the cytoplasm and subjected to ubiquitinated degradation, thus leading to the reduced expression of genes that are involved in gluconeogenesis, such as glucose-6-phosphatase (G6Pase) and phosphoenolpyruvate carboxykinase 1 (Pepck) (reviewed in refs. 24, 25). Full activation of AKT requires phosphorylation at 2 sites, T308 and S473. AKT-T308 is mainly phosphorylated by PDK1 $(26,27)$. A recent study showed that AKT-S473 is specifically phosphorylated by the mammalian target of rapamycin complex 2 (mTORC2) (28). However, the functional relevance of AKT-S473 phosphorylation in regard to FOXO1 phosphorylation and gluconeogenesis has not been established.

In this study, we investigated the effect of SIRT1 deficiency on gluconeogenesis in the Sirt1 $1^{\text {exon5-6/exons-6; }}$ Alb-Cre mice (22) and provide evidence that SIRT1 negatively regulates gluconeogenesis. We demonstrate that SIRT1 positively regulates expression of Rictor, a key component of the mTORC2 complex (29). The reduced Rictor levels in SIRT1 mutant mice impaired phosphorylation of AKTS473, caused hypophosphorylation of FOXO1-S253, and increased expression of G6pase and Pepck, which, in turn, resulted in chronic hyperglycemia and widespread oxidative stress and, eventually, whole-body insulin resistance under regular feeding conditions.

\section{Results}

Liver-specific deletion of SIRT1 results in byperglycemia due to increased gluconeogenesis. To investigate the role of SIRT1 in gluconeogenesis, we first measured blood glucose levels in 1- to 2-month-old mice and detected significantly higher levels of blood glucose in Sirt1 liver-specifc knockout ( $\operatorname{Sirt}^{\mathrm{LKO}}$ ) animals than in controls in both fed and fasting conditions (Figure 1A and data not shown). Next, 


\section{A}

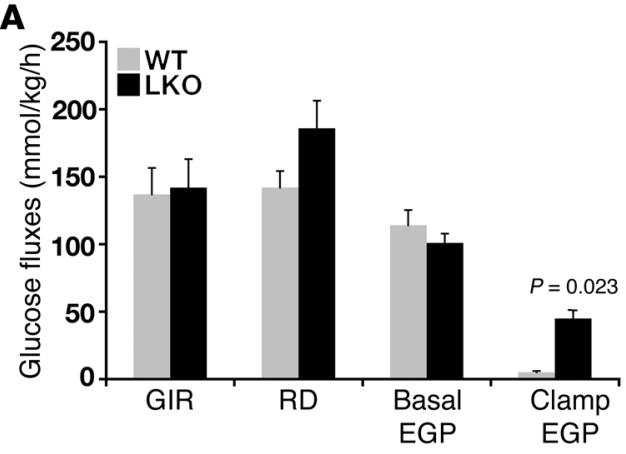

C
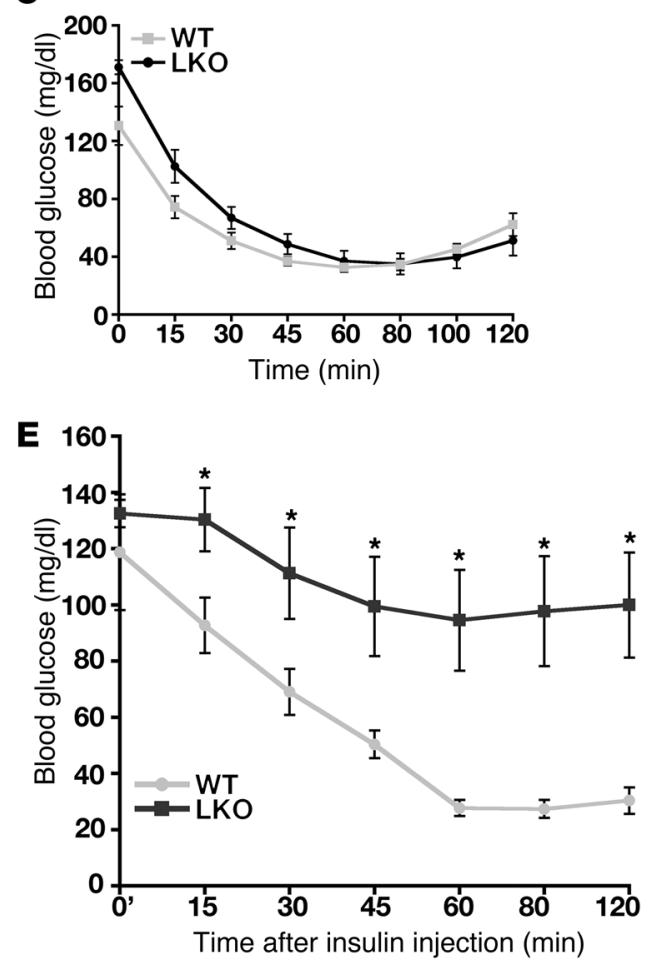

B
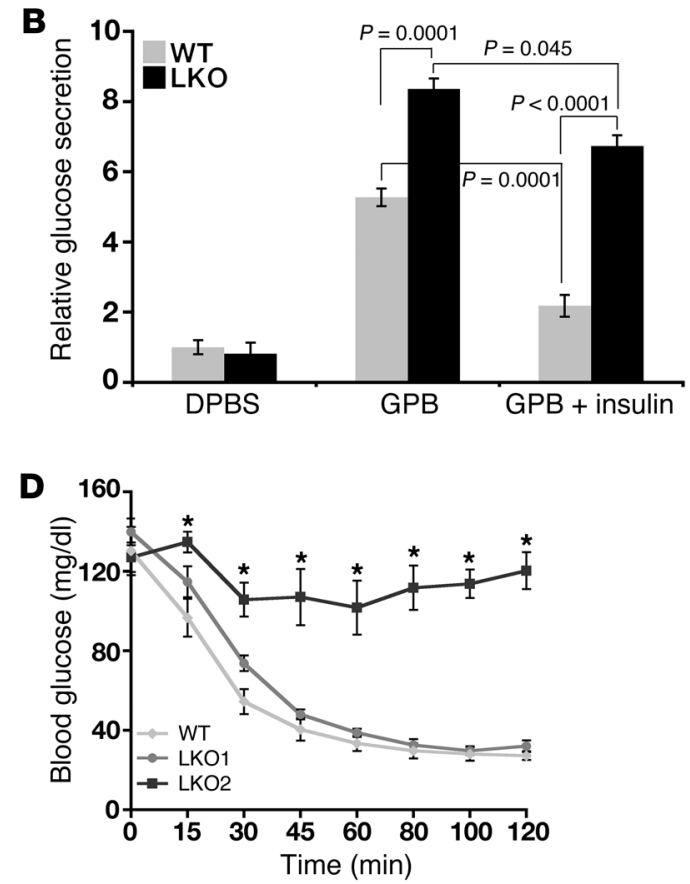

$\mathbf{F}$

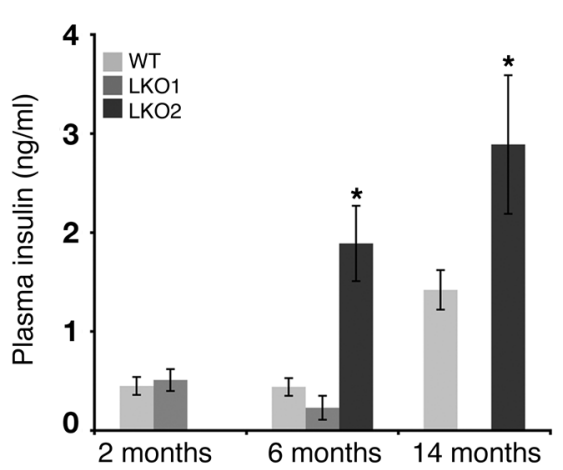

Figure 2

Liver-specific deletion of SIRT1 leads to insulin resistance. (A) The livers of Sirt1LKO mice are insulin resistant. Hyperinsulinemic-euglycemic clamp experiment of 6 control and 7 mutant mice at 2 months of age. Rd, whole-body glucose disposal rate; Basal EGP, basal endogenous glucose production; Clamp EGP, endogenous glucose production during clamp. (B) Primary hepatocytes from Sirt1LKo liver produce more glucose and are resistant to insulin treatment $(100 \mathrm{nM})$ compared with the hepatocytes from wild-type liver $(n=3)$. Sirt 1 LKO and wild-type cells exhibit about a 2-fold difference in response to insulin treatment: glucose secretion drops to $41 \%$ in wild-type cells $(P=0.0001)$ and to $80 \%$ in Sirt1 ${ }^{\text {LKO }}$ cells $(P=0.045)$. GBP, glucose production buffer. (C) ITT was performed with 2-month-old Sirt1LKO mice ( $n \geq 9$ pairs). (D) ITT was performed with 6-month-old Sirt1 ${ }^{L K O}$ mice. Ten wild-type mice and fifteen Sirt1 ${ }^{L K O}$ mice were analyzed. Five Sirt1 ${ }^{L K O}$ mice (LKO2) displayed ITT resistance; ten Sirt1LKO mice (LKO1) were still sensitive to insulin challenge. ${ }^{*} P<0.01$. (E) ITT performed in 14 -month-old mice $(n=9)$. ${ }^{*} P<0.01$. (F) Plasma insulin levels of mice at 2 months (15 pairs), 6 months (16 pairs), and 14 months (16 pairs) of age. All Sirt 1 LKO mice at 2 months of age and 10 Sirt1LKO (LKO1) mice at 6 months of age displayed normal insulin content. These mice belong to the LKO1 group. Six Sirt ${ }^{L K O}$ mice at 6 months of age and all Sirt ${ }^{L K O}$ mice at 14 months of age contain higher plasma insulin levels than controls $\left({ }^{\star} P<0.02\right)$. These mice belong to the LKO2 group.

we performed a pyruvate tolerance test (PTT) to determine whether the increased blood glucose in Sirt $1^{L K O}$ mice was due to increased hepatic glucose production. We found that Sirt $1^{L K O}$ mice had higher blood glucose levels after pyruvate administration, suggesting increased hepatic glucose production (Figure 1B). Consistent with the increased hepatic glucose production, the expression levels of gluconeogenetic genes, G6pase and Pepck, were increased by 2 to 3 fold at both mRNA and protein levels (Figure 1, C and D). Sirt $1^{\text {LKO }}$ mice also displayed glucose intolerance during a glucose tolerance test (GTT) (Figure 1E). We have studied insulin secretion of Sirt ${ }^{\text {LKO }}$ mice and did not detect a significant difference in insulin content during the GTT between control and Sirt $1^{\text {LKO }}$ mice (Figure 1F).

SIRT1 deficiency leads to bepatic insulin resistance and gradually results in whole-body insulin resistance in older mice. Under normal physiological conditions, the liver's response to insulin is the key factor in shutting down glucose production by inhibiting transcription of Pepck 


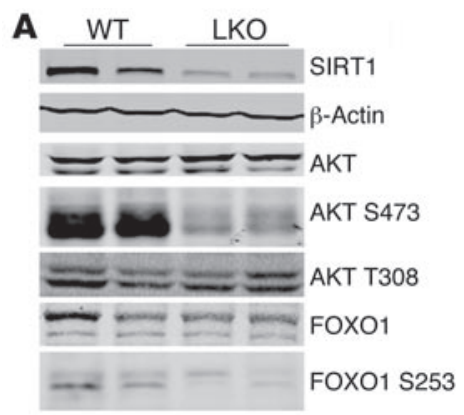

B
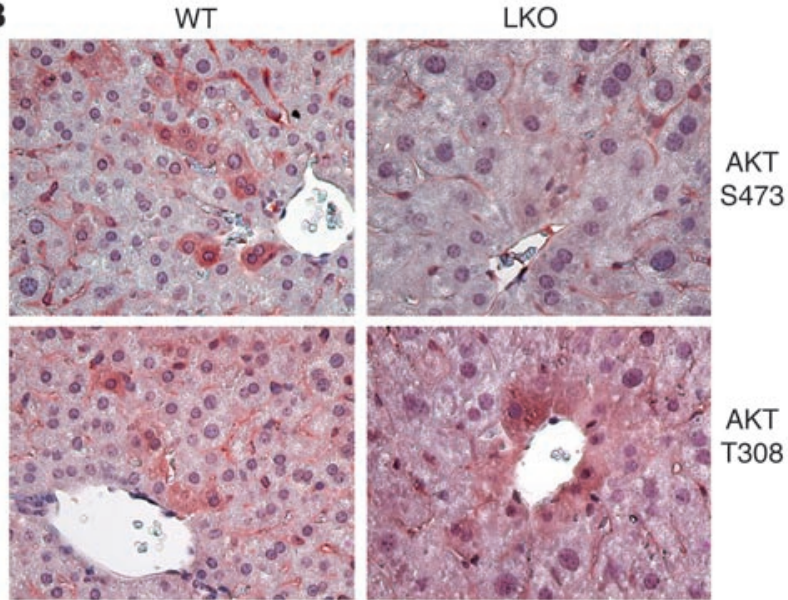

C

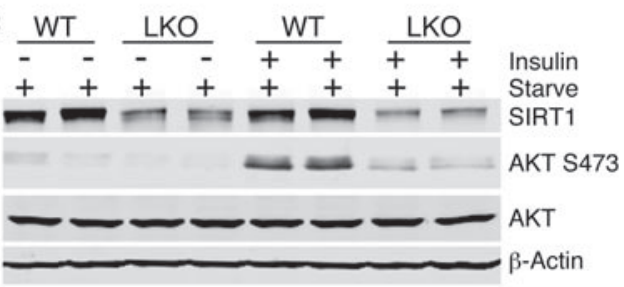

D

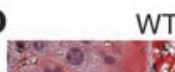

WT
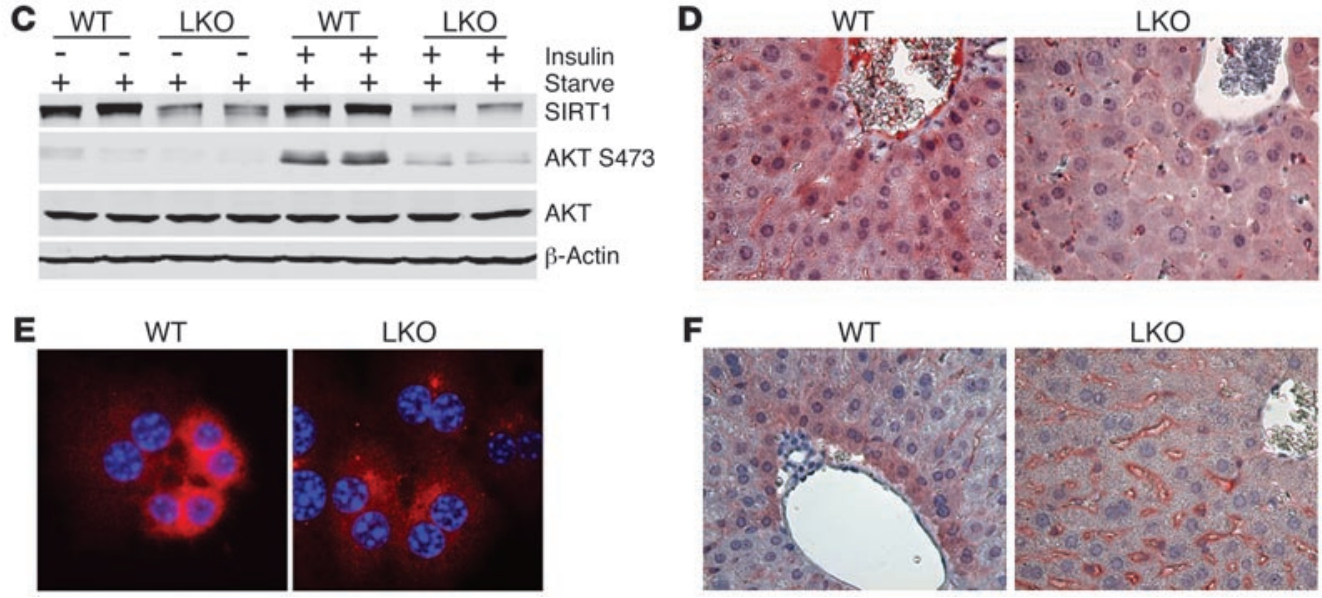

$\mathbf{F}$

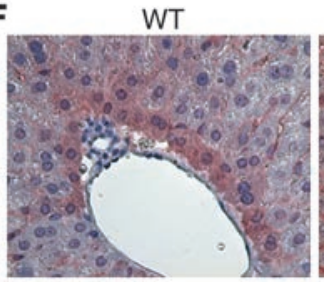

LKO

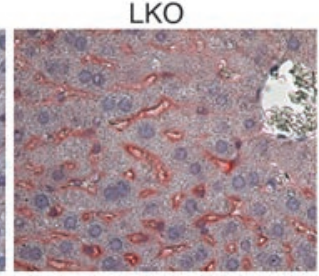

Figure 3

Deletion of SIRT1 reduces the phosphorylation level of AKT-S473. (A) Western blots show decreased pAKT-S473 in Sirt1 ${ }^{\text {LKO }}$ liver under regular fed condition. (B) IHC staining confirms the lack of S473 phosphorylation in Sirt1LKO liver. (C) Western blots demonstrate decreased pAKT-S473 in Sirt1LKO liver in response to insulin injection. (D) IHC staining displays the reduced level of pAKT-S473 in Sirt1 ${ }^{\text {LKO }}$ liver upon insulin stimulation. (E) Immunofluorescent staining shows that in the primary hepatocytes from Sirt 1 LKO liver, FOXO1 phosphorylation and cytoplasmic translocation did not occur upon insulin stimulation. The primary hepatocytes were stained with antibody against phosphorylated FOX01-S253. (F) IHC reveals impaired phosphorylation of FOXO1-S253 in Sirt1LKO liver that is stimulated by insulin. Original magnification, $\times 130$ (B, D, and F); $\times 260$ (E). and G6pase after a meal $(30,31)$. To investigate whether liver-specific deletion of SIRT1 causes hepatic insulin resistance, we performed euglycemic-hyperinsulinemic clamps in 2-month-old Sirt ${ }^{\text {LKO }}$ mice and wild-type control mice. The Sirt $1^{L K O}$ mice were morphologically normal and had similar body weight compared with that of controls (data not shown). The clamps indicated that the glucose infusion rate (GIR) required to maintain blood glucose at constant levels during the clamp was not different between $\operatorname{Sirt} 1^{L K O}$ and control mice, indicating comparable insulin sensitivity (Figure $2 \mathrm{~A}$ and Supplemental Figure 1, A-C; supplemental material available online with this article; doi:10.1172/JCI46243DS1). SIRT1 mutant and control mice also showed no significant differences in the wholebody glucose disposal rate (Figure 2A) and 2-deoxy-glucose uptake into multiple tissues, including muscle, adipose tissue, and brown fat (Supplemental Figure 1, D and E). These observations suggest that at 2 months of age the whole-body insulin sensitivity was normal in the Sirt ${ }^{L K O}$ mice. Insulin tolerance test (ITT) also revealed no difference between SIRT1 mutant and wild-type mice (Figure 2C). However, under the clamp condition, Sirt $1^{L K O}$ mice showed significantly increased endogenous glucose production (clamp EGP; Figure $2 \mathrm{~A}$ ), suggesting hepatic insulin resistance. Consistently, primary hepatocytes from Sirt $1^{\text {LKO }}$ liver produced about 2 -fold more glucose than those from wild-type liver and exhibited resistance to insulinmediated inhibition of glucose production (Figure 2B). Altogether, these data indicate that the impact of SIRT1 loss is largely restricted to the liver at this developmental stage.
To investigate whether the older SIRT1 mutant animals developed whole-body insulin resistance, we performed ITT on 6-month-old and 14-month-old animals. At 6 months of age, among 15 mutants tested, 5 mutants (LKO2 group) displayed insulin resistance, i.e., failed to achieve a $50 \%$ or more reduction in blood glucose by 45 minutes after insulin administration, as exhibited by the remaining 10 mutants (LKO1 group) and wild-type animals (Figure 2D). However, at 14 months of age, all SIRT1 mutant animals tested $(n=9)$ displayed insulin resistance (Figure 2E). These data indicate that SIRT1 mutant mice gradually developed insulin resistance when they were getting older. Consistent with the insulin resistance, Sirt ${ }^{L K O}$ mice exhibited much higher plasma insulin levels than control mice, starting from 6 months of age (Figure 2F). These observations underscore an essential role of SIRT1 in maintaining the normal functions of the liver in glucose production and insulin sensitivity. Impaired hepatic function of SIRT1 first causes increased glucose production in the liver, leading to hyperglycemia, which eventually results in whole-body insulin resistance in older mice.

SIRT1 deficiency impairs AKT activity through reducing AKT phosphorylation at $\$ 473$. Next, we investigated possible causes for the increased expression of G6pase and Pepck genes. It has been shown that insulin represses most of the hepatic genes, and this effect is mediated through a phosphoinositide 3-kinase (PI3K) pathway, in which insulin activates PI3K, resulting in phosphorylation of AKT (32-34). A previous study also detected a reduced level of pAKTS473 in Sirt1 exon4/exon4; Alb-Cre mice fed with high-fat diet (20). To 

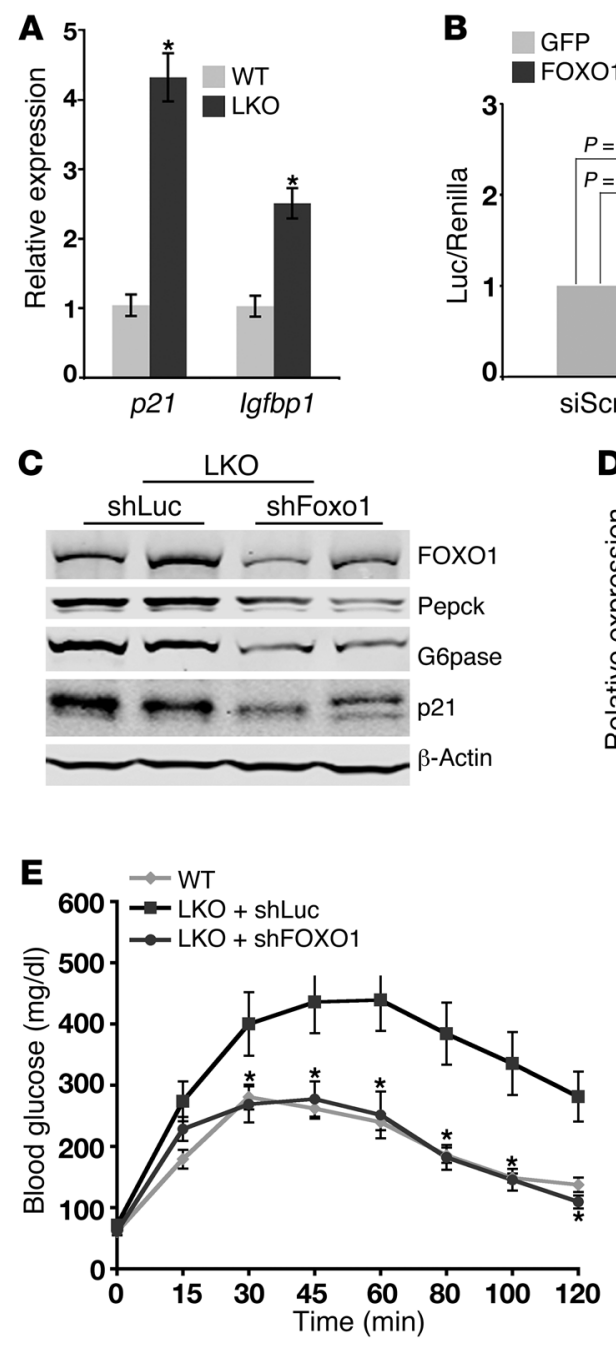

B
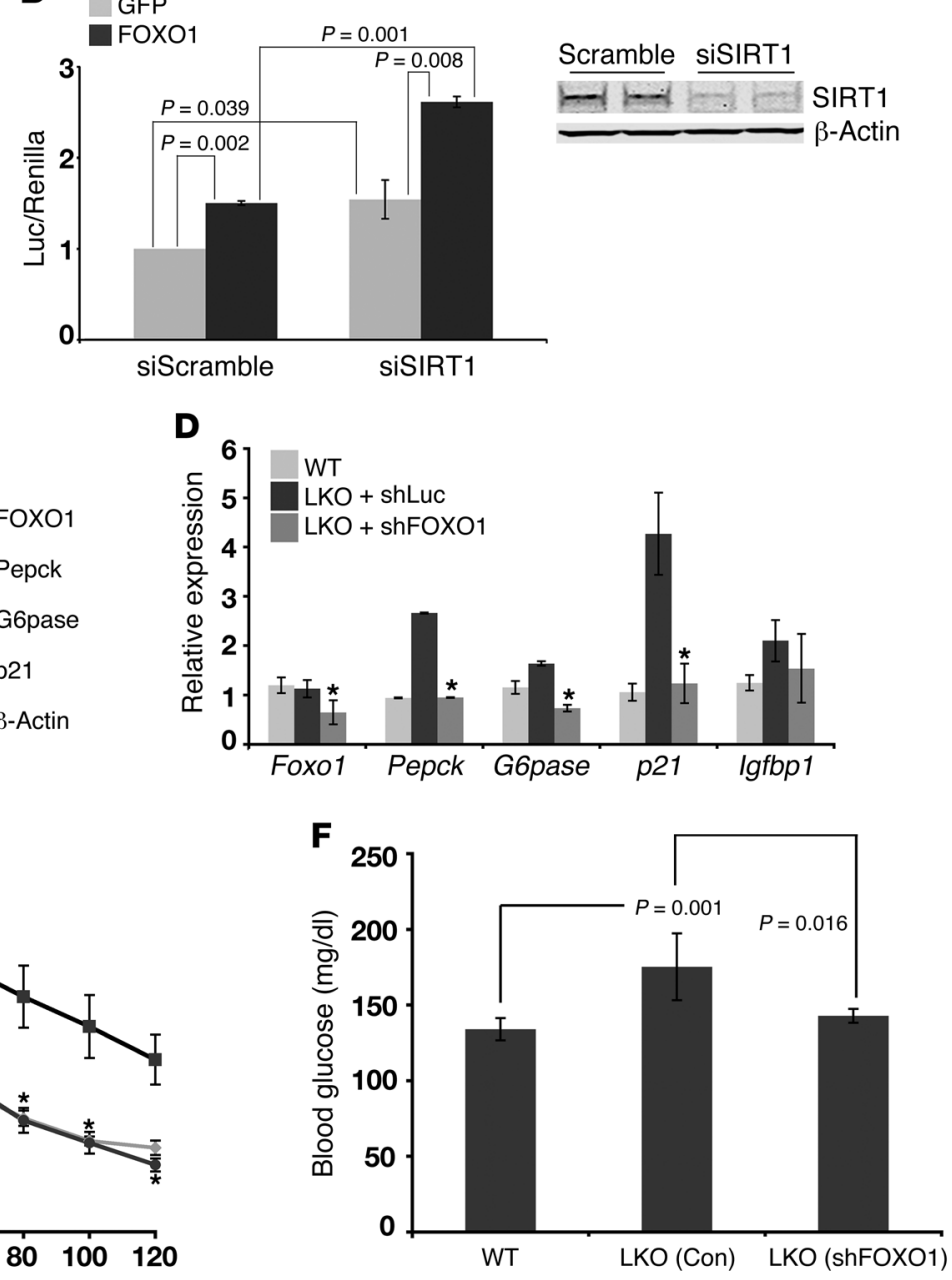

\section{Figure 4}

FOXO1 reduction in Sirt1LKO liver corrected hepatic glucose overproduction. (A) qRT-PCR demonstrates that the expression of FOXO1 downstream genes, $p 21$ and Igfbp1, was increased in Sirt1 $\angle K O$ liver. ${ }^{*} P<0.01$. (B) Ectopic overexpression of FOXO1 increases transcriptional activity of the Pepck promoter. This effect is enhanced by knockdown of SIRT1. Hepa1-6 cells were transfected with Pepck-luc, together with a FOXO1 expression vector (FOXO1) or a GFP expression vector as a control (GFP). The scramble siRNA oligos or SIRT1-specific siRNA oligos were in combination with the GFP and Foxo1 transfection. The blot shows the knockdown level of SIRT1. (C and D) shRNA knockdown of FOXO1 (shFOXO1) in Sirt1LKO mouse liver reduced expression of G6pase, Pepck, and p21 at both (C) protein and (D) mRNA level. (E) shRNA knockdown of FOXO1 in Sirt1 ${ }^{\text {LKO }}$ mice restored their ability to respond to glucose challenge. ${ }^{*} P<0.0001$. (F) shRNA knockdown of FOXO1 in liver reduced blood glucose level in Sirt1 ${ }^{L K O}$ mice. Con, shLuc control.

study whether SIRT1 deficiency could also affect pAKT under regular fed condition in our mutant mice, we performed Western blot analyses using antibodies against pAKT-T308 and pAKT-S473, 2 residues that are commonly phosphorylated by upstream signaling (28). Our data detected a marked reduction of pAKT-S473, while no change was detected on pAKT-T308 (Figure 3A). Direct staining of liver sections confirmed that mutant hepatocytes contained low levels of pAKT-S473, while the intensity of PAKT-T308 was comparable with that of wild-type liver (Figure 3B). Consistent with this, downstream targets of pAKT-T308, such as PGSK3 and p70S6K, were not affected (Supplemental Figure 2).

It is known that insulin stimulates phosphorylation of AKT in mouse liver. To test the insulin response, insulin was injected into control and mutant animals that were fasted for 6 hours, and the livers were collected 30 minutes after injection. We found that insulin was able to stimulate the phosphorylation of AKT-S473 in wild-type liver, but it failed to do so in mutant livers (Figure 3C). Immunohistochemistry (IHC) on the above liver sections demonstrated that mutant livers displayed markedly reduced levels of pAKT-S473 compared with those of controls (Figure 3D), while no change in PAKT-T308 was observed (data not shown).

AKT regulates gluconeogenesis through affecting the stability of FOXO1, as AKT directly phosphorylates FOXO1 at S253, leading to its nuclear exclusion $(35,36)$. We then checked the phosphorylation status of FOXO1 in fed livers by Western blot analysis and IHC. Western blot analysis revealed that the phosphorylation level of FOXO1-S253 was dramatically reduced in the livers of Sirt1 ${ }^{\text {LKO }}$ mice (Figure 3A). Insulin-induced phosphorylation of FOXO1- 
A

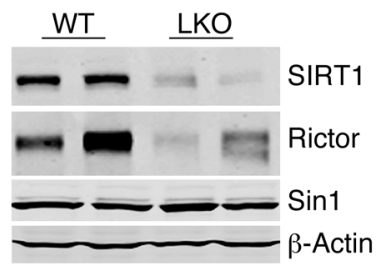

B

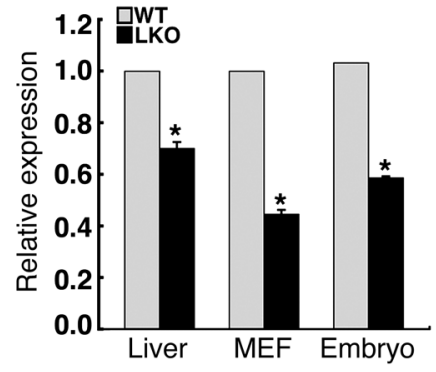

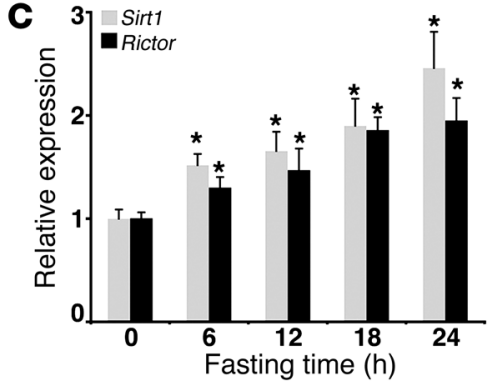
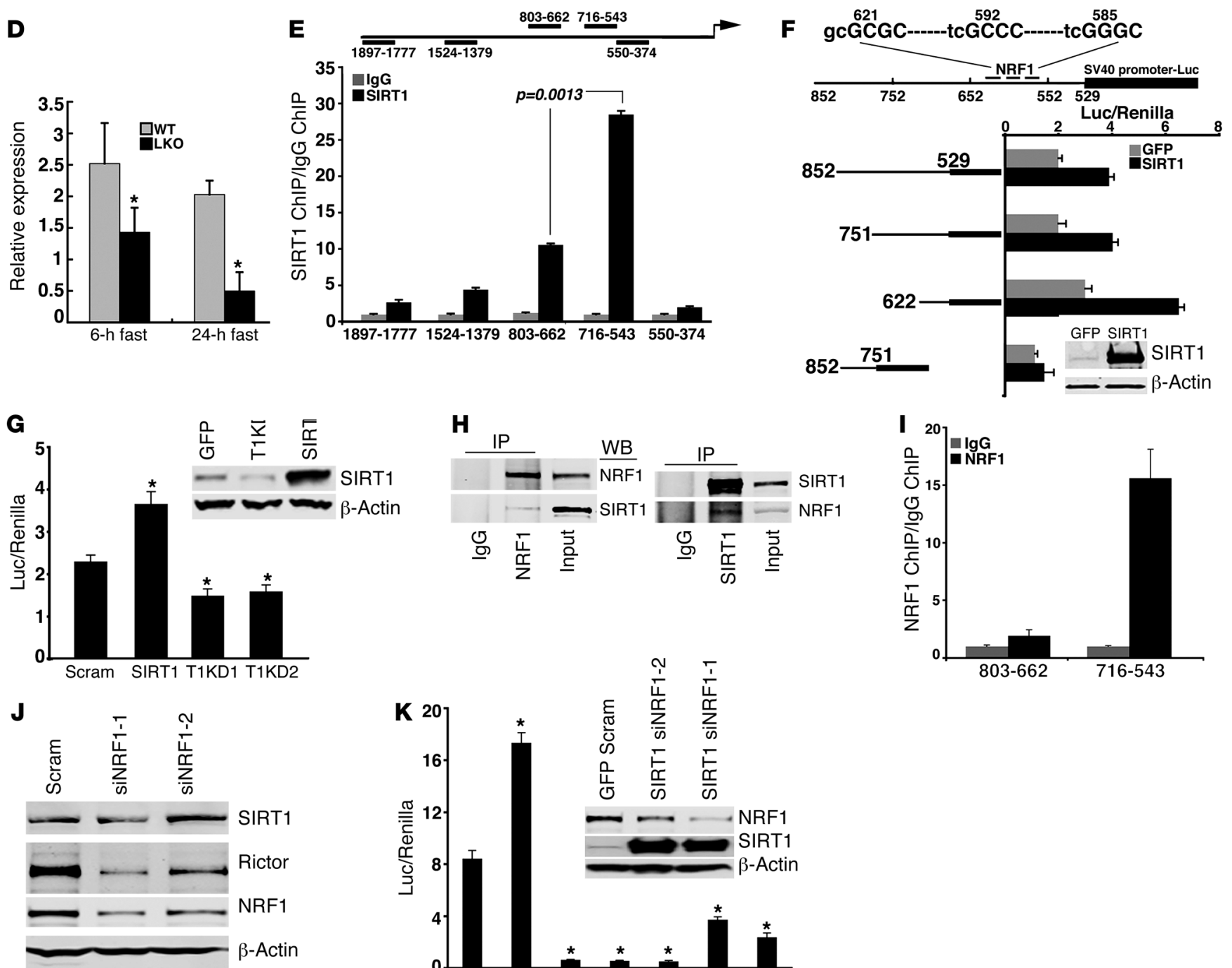

Figure 5

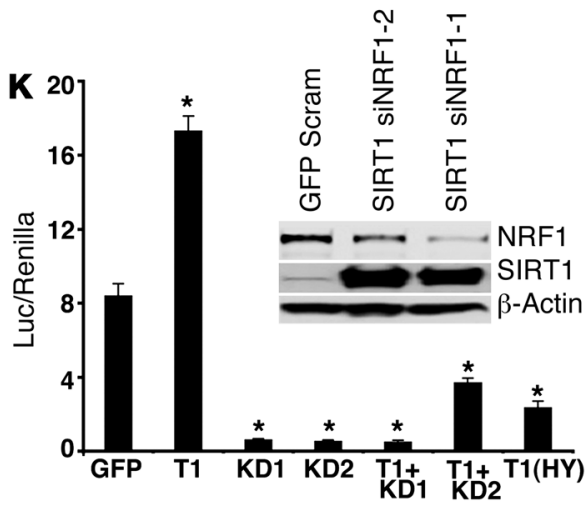

SIRT1 positively regulates Rictor expression in the liver. (A) Western blotting shows reduced Rictor in Sirt1LKO liver. (B) Real-time RT-PCR analysis reveals that Rictor is reduced in Sirt1 ${ }^{L K O}$ liver, SIRT1-null MEFs, and embryos. ${ }^{*} P<0.001$. (C) Rictor is upregulated upon fasting in close correlation with Sirt1. ${ }^{*} P<0.05$, when compared with 0 hour. (D) The increase of Rictor level is impaired in Sirt1LKO liver under 6-hour or 24-hour fasting conditions. Six mice (2 to 3 months of age) were used at each time point. ${ }^{*} P<0.001$. (E) ChIP assay shows that SIRT1 binds to 2 fragments in the promoter of Rictor upstream of ATG, 803-662 bp and 716-543 bp. The diagram displays the location of all primers used. (F) Serial deletion assay of Rictor promoter-pGL3B demonstrates that SIRT1 strongly activates the fragment that contains the NRF1-binding site. The diagram shows 3 potential core NRF1-binding sites. The blot shows SIRT1 levels in the GFP- and SIRT1-transfected cells. (G) SIRT1 overexpression upregulates the luciferase activity of 622-529-pGL3B. Acute knockdown of SIRT1 by 2 different shRNA constructs specific to SIRT1 (T1KD1, T1KD2) reduces luciferase activity of 622-529-pGL3B. The blot displays the level of SIRT1 under overexpression (SIRT1) or knockdown (T1KD) conditions. ${ }^{*} P<0.001$. (H) Reciprocal immunoprecipitation reveals that endogenous SIRT1 and NRF1 interact with each other. Input: $5 \%$ of total protein. (I) ChIP assay with NRF1 antibody demonstrates that NRF1 binds to predicted NRF1-binding sites. (J) NRF1 acute knockdown by 2 shRNA constructs specific to NRF1 (KD1, KD2) decreased endogenous Rictor level. (K) NRF1 acute knockdown by 2 shRNA constructs specific to NRF1 greatly reduces luciferase activity of 622529-pGL3B. This effect cannot be overridden by ectopic expression of SIRT1 (T1). ${ }^{*} P<0.001$. 
$\mathbf{A}$

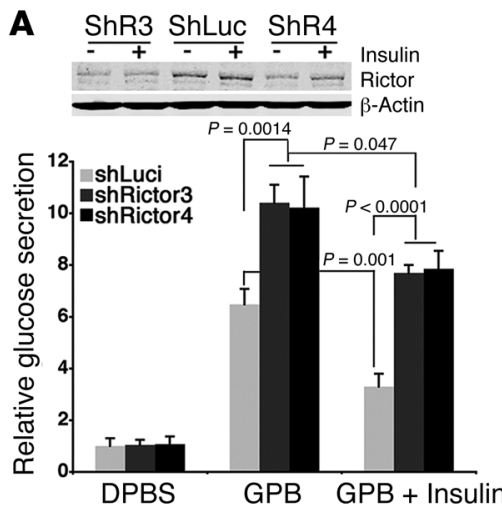

\section{D}

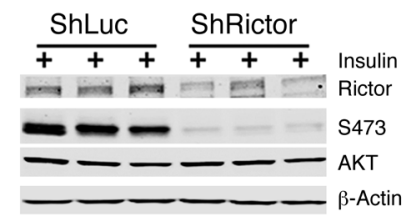

E

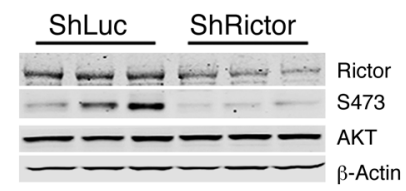

H

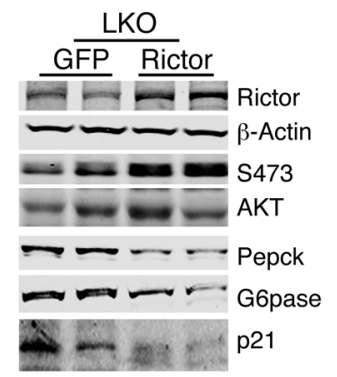

B

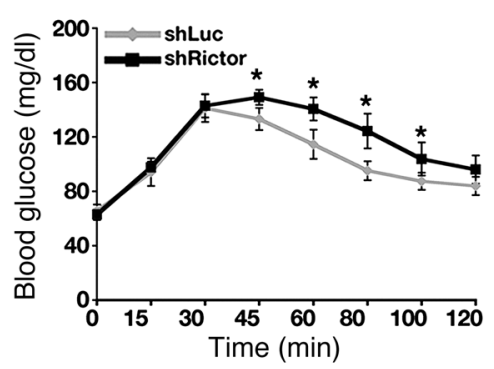

F

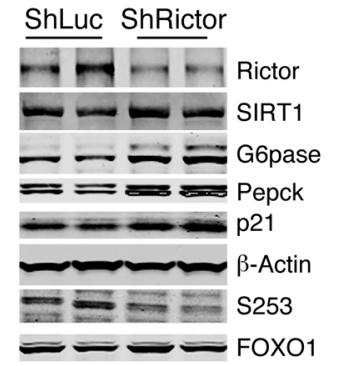

C

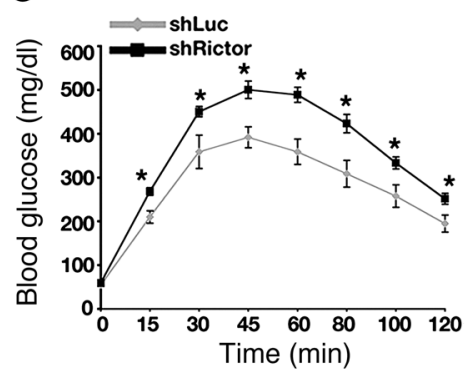

G
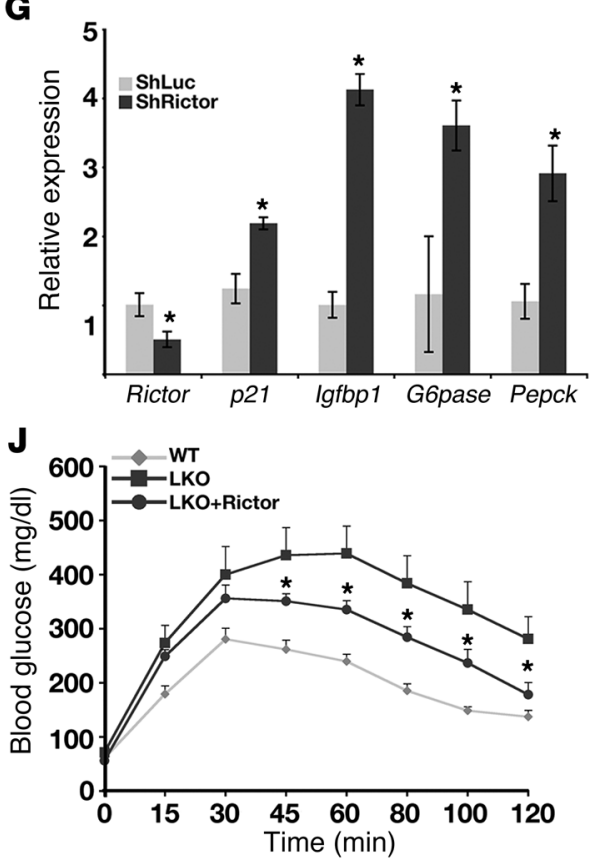

\section{Figure 6}

Rictor knockdown greatly increases hepatic gluconeogenesis. (A) Acute knockdown of Rictor in wild-type primary hepatocytes leads to glucose overproduction and insulin resistance. Rictor wild-type and Rictor acute knockdown cells exhibit about a 1.5-fold difference in response to insulin treatment: glucose secretion drops to $51 \%$ in wild-type cells $(P=0.0001)$ and to $75 \%$ in cells carrying the shRNA-mediated knockdown of Rictor $(P=0.047)$. The blot shows Rictor levels. (B) Acute knockdown of Rictor in wild-type mouse liver causes PTT intolerance. Three-month-old wildtype male mice were injected with shLuciferase virus or shRictor virus $\left(n=9\right.$ each group). ${ }^{*} P<0.05$. (C) Mice with acute knockdown of Rictor display glucose intolerance assessed by GTT $(n=9)$. ${ }^{*} P<0.02$. (D and E) Western blot analysis shows the reduction of total Rictor level and decreased AKT S473 phosphorylation due to acute Rictor knockdown in response to (D) insulin stimulation or (E) under normal fed conditions. ( $\mathbf{F}$ and $\mathbf{G}$ ) Liver samples from control and Rictor knockdown mice were used for (F) Western blot and (G) qRT-PCR analysis to demonstrate reduction of total Rictor level and the alteration of downstream genes due to shRNA injection. ${ }^{\star} P<0.01$. (H-J) In SIRT1LKO mice, overexpressing Rictor improved glucose intolerance status. Three-month-old SIRT1LKO mice were injected with shLuciferase virus or Rictor-overexpressing virus ( $n=10$ each). (H) Western blots and (I) qRT-PCR reveal overexpression of Rictor and its downstream responders. ${ }^{*} P<0.01$. (J) Overexpressing Rictor improved the GTT in SIRT1LKO mice. ${ }^{*} P<0.05$.

S253 and its cytoplasm distribution were also observed in wildtype hepatocytes, but not in SIRT1 mutant primary hepatocytes, as revealed by immunofluorescent staining with an antibody against pFOXO1-S253 (Figure 3E). This result was confirmed by IHC staining of pFOXO1-S253 on liver sections (Figure 3F).

Knockdown of FOXO1 reverted the phenotype of Sirt $1^{1 K O}$ mice. Reduced phosphorylation of pFOXO1-S253 suggests a higher activity of FOXO1 in SIRT1 mutant liver. To investigate this, qRT-PCR was performed to detect the expression of FOXO1 downstream genes, such as $p 21$ and $I g f b p 1$ (35). Indeed, expression of these genes was significantly higher in the mutant liver than in the control liver (Figure 4A), confirming that in SIRT1 mutant livers, FOXO1 is more active. FOXO1 positively regulates transcription of G6pase and Pepck genes $(37,38)$. Using a luciferase reporter construct for the Pepck gene, we showed that ectopic expression of FOXO1 activated the Pepck promoter in HepG2 cells (Figure 4B). Consistent with our data that 

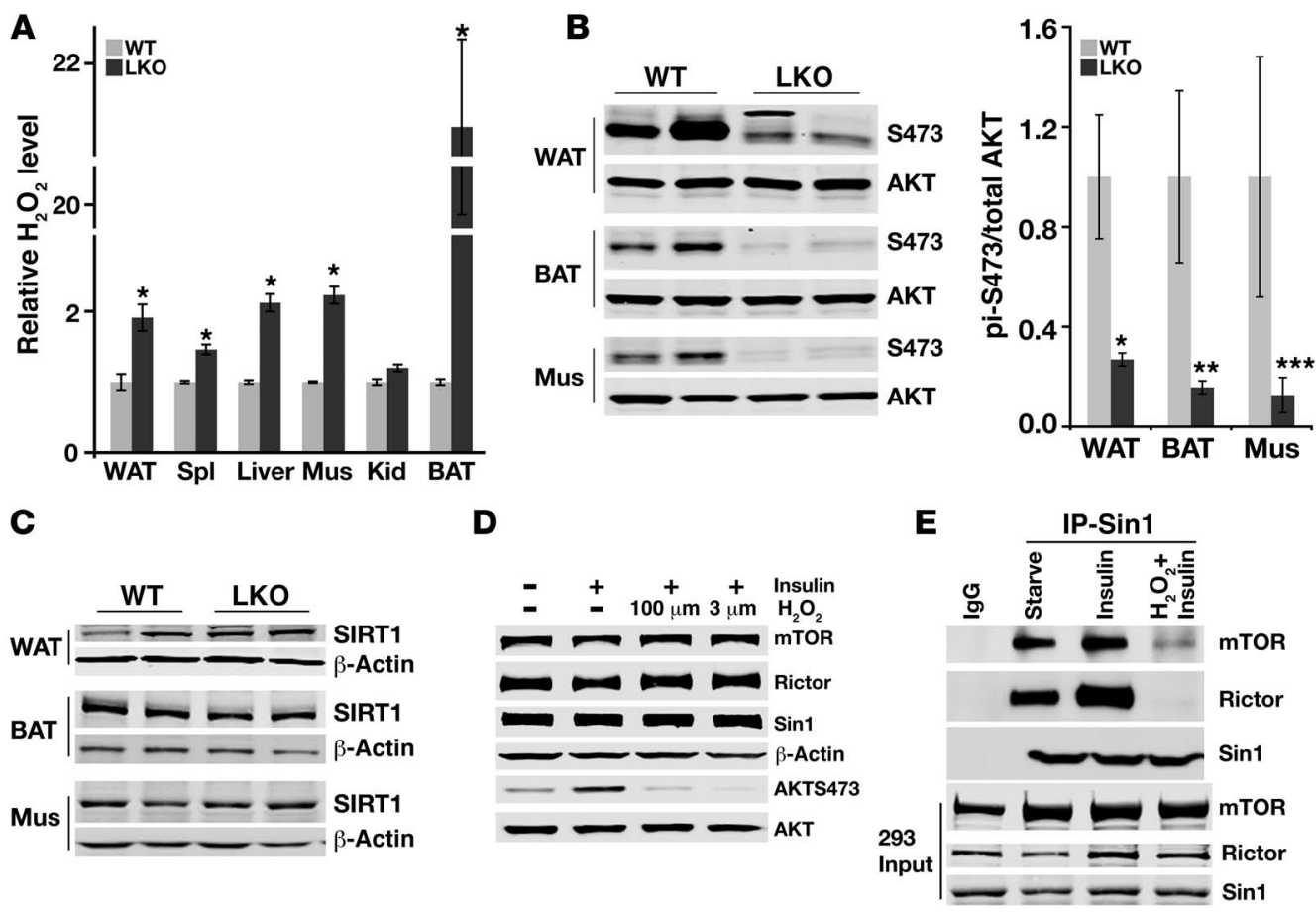

$\mathbf{F}$

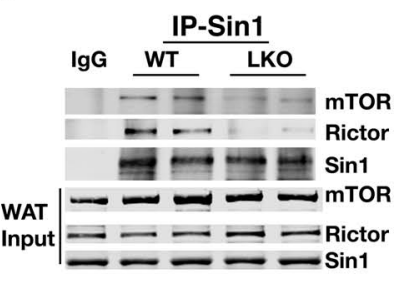

Figure 7

Insulin resistance in Sirt1LKO mice is associated with elevated intracellular ROS that can be reversed by antioxidants. (A) Sirt1LKO mice have higher $\mathrm{H}_{2} \mathrm{O}_{2}$ levels than those of controls. Twelve pairs of 18 -month-old mice were used. ${ }^{*} P \leq 0.01$. (B) Western blot analysis reveals consistently decreased pAKT-S473 in white adipose tissue (WAT), BAT, and muscle (mus) tissues of 18-month-old Sirt1LKO mice. pi-S473, pAKT-S473. The bar graph on the right reveals the quantification of $S 473$ phosphorylation over total AKT. ${ }^{\star} P=0.02 ;{ }^{\star \star} P=0.0039 ;{ }^{* \star \star} P=0.011$. (C) In old mice, SIRT1 levels do not change between WT and Sirt1LKO mice. (D) In 293HEK cells, $\mathrm{H}_{2} \mathrm{O}_{2}$ treatment blocks the induction of pAKT-S473 by insulin. The cells were starved for 12 hours, followed by treatment with $100 \mu \mathrm{M} \mathrm{H}_{2} \mathrm{O}_{2}$ for 12 hours or $3 \mathrm{mM} \mathrm{H}_{2} \mathrm{O}_{2}$ for 1 hour prior to insulin (100 $\mathrm{nM}$ ) addition for 30 minutes. (E) In 293 cells, $\mathrm{H}_{2} \mathrm{O}_{2}$ treatment impaired the complex formation of mTOR, Rictor, and Sin1. Immunoprecipitation with an antibody against $\mathrm{Sin} 1$ demonstrates that less mTOR and Rictor are associated with $\mathrm{Sin} 1$ in the presence of $\mathrm{H}_{2} \mathrm{O}_{2}$. $(\mathbf{F})$ In WAT tissue from 18-month-old Sirt1 ${ }^{L K O}$ mice, the interaction among mTOR, Rictor and Sin1 was decreased. Immunoprecipitation was carried out as in $\mathbf{D}$.

SIRT1 deficiency increases FOXO1 activity, knockdown of SIRT1 by siRNA, together with overexpression of FOXO1, synergistically elevated the Pepck promoters (Figure 4B). Similar data were observed in the G6pase promoter (data not shown). Together with our finding that SIRT1 mutant liver has increased levels of G6pase and Pepck, we believe that the increased FOXO1 activity is responsible for the enhanced gluconeogenesis in SIRT1 mutant liver.

To provide further proof for this notion, we performed FOXO1 knockdown in the livers of Sirt $1^{L K O}$ mice by injecting lentivirus that expresses shRNA against FOXO1 through the tail vein at a dose of $1 \times 10^{8}$ viruses in $200 \mu \mathrm{l}$ PBS per mouse. Our data indicated that knockdown of FOXO1 in Sirt ${ }^{L K O}$ liver reverted the overexpression of FOXO1 downstream genes, including p21, G6pase, and Pepck, measured by Western blot (Figure 4C) and qRT-PCR (Figure 4D). It also restored the response of Sirt $1^{L K O}$ mice to glucose challenge (Figure 4E) and normalized the glucose level (Figure 4F).

SIRT1 positively regulates expression of Rictor, a component of $m$ TORC2 complex. AKT-S473 is phosphorylated by the mTORC2 complex in response to growth factor stimulation (39). Our study indicated that one of mTORC2 components, Rictor, was downregulated in the $\operatorname{Sirt} 1^{L K O}$ liver, while another component, $\operatorname{Sin} 1$, was not altered (Figure 5A). The decreased expression of Rictor was also detected at mRNA level in SIRT1 mutant liver $(P<0.001)$, embryos $(P<0.001)$, and mouse embryonic fibroblasts (MEFs) $(P<0.001)$ compared with that in wild-type controls (Figure 5B). These observations prompted us to hypothesize that SIRT1 plays a positive role in regulating Rictor expression. To investigate this, we performed the following further experiments.

We first performed a time-course study of Sirt1 and Rictor expression in the liver after fasting. We detected significantly increased expression of both genes 6 hours after fasting, and there is a positive correlation of SIRT 1 induction and Rictor induction at multiple points thereafter (Figure 5C), while SIRT1 deficiency abolished Rictor induction (Figure 5D). Fasting-induced Rictor expression was confirmed in 2 cell lines, HepaG2 and Hepa1-6, which were derived from liver (Supplemental Figure 3A). The induction of Rictor expression is correlated with increased SIRT1 expression, as we found that the similar fast condition failed to induce SIRT1 and also could not induce Rictor expression in HEK293 cell line, which was derived from kidney (Supplemental Figure 3A). We further showed that overexpression of SIRT1 in these cell lines elevated the Rictor level (Supplemental Figure 3B), while knockdown of SIRT1 by siRNA markedly reduced it (Supplemental Figure 3C).

Next, we investigated the potential mechanism underlying the regulation of SIRT1 to Rictor expression. ChIP analysis with SIRT1 antibody detected the existence of SIRT1 on 2 fragments upstream of Rictor ATG, 803-662 bp and 716-543 bp, with the latter having much stronger binding to Sirt 1 than the former (Figure 5E). We 

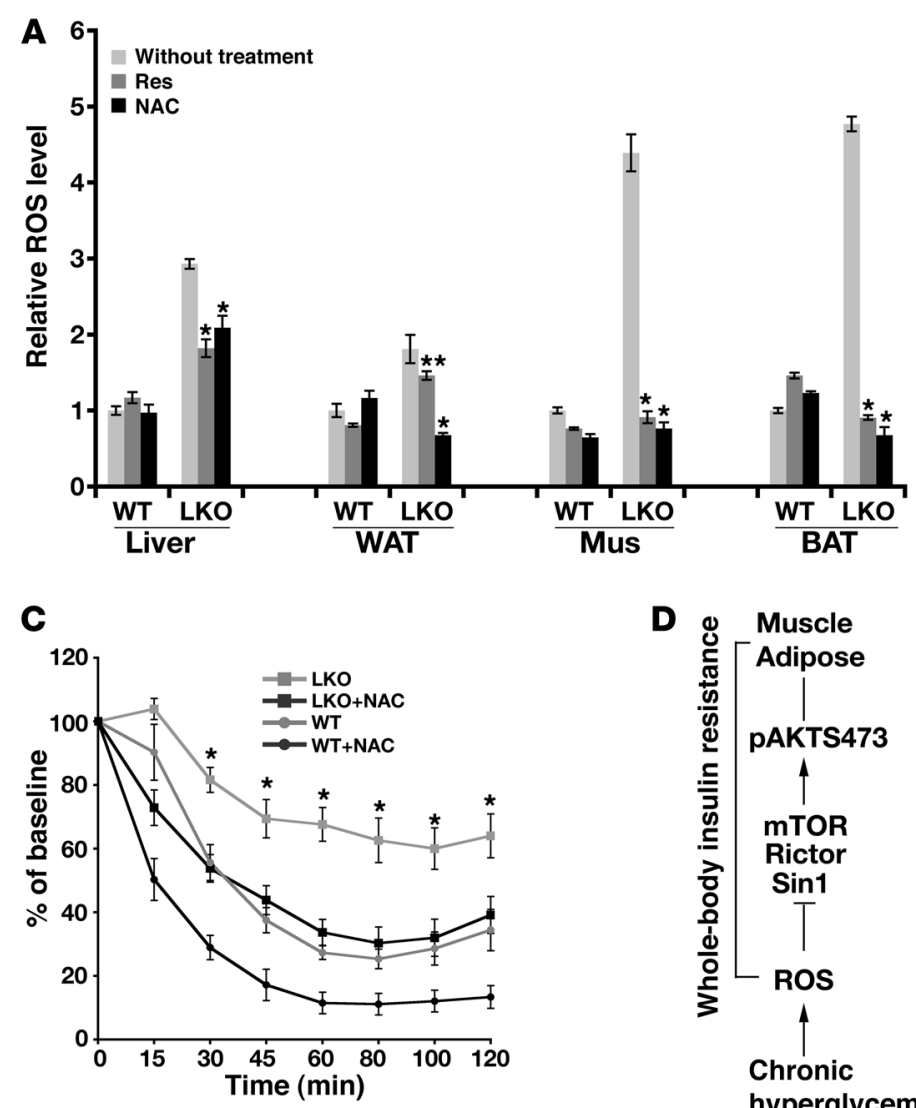
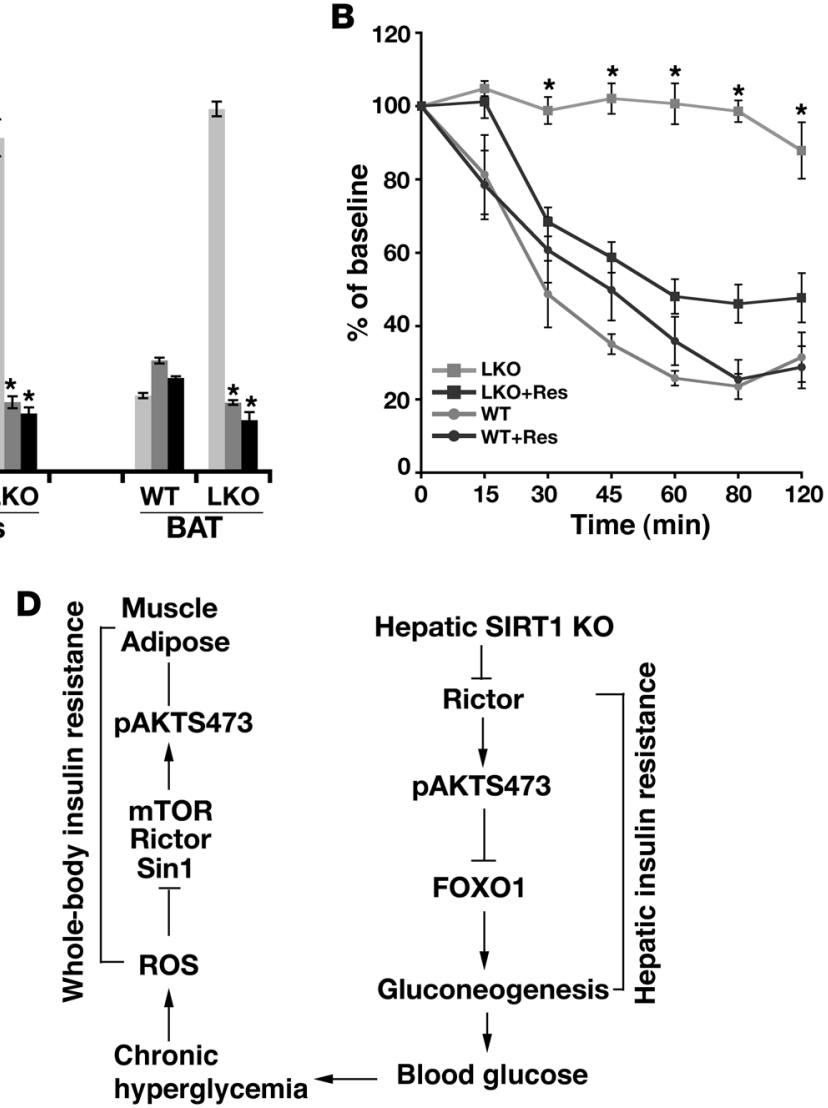

\section{Figure 8}

Antioxidant treatment improved insulin sensitivity in old Sirt1LKO mice. (A) Resveratrol $(0.8 \mathrm{mg} / \mathrm{ml}$ for 9 weeks) and NAC (1 g/l for 9 weeks) treatments significantly lowered ROS level in multiple tissues. ${ }^{*} P<0.01 ;{ }^{* *} P<0.05$. (B) Resveratrol $(0.8 \mu \mathrm{g} / \mathrm{ml}$ for 9 weeks) treatment improves the ITT response significantly in 18-month-old Sirt 1 LKO mice ( $n=7$ per group). ${ }^{\star} P<0.001$. (C) NAC (1 g/l for 9 weeks) treatment improves the ITT response in 12-month-old Sirt1 LKO mice ( $n=12$ per group). ${ }^{*} P<0.01$. ITT in $\mathbf{B}$ and $\mathbf{C}$ was performed before and after treatment. (D) A model showing that hepatic disruption of SIRT1 induces whole-body insulin resistance through increasing blood glucose and ROS in insulin-sensitive organs/tissues.

showed that ectopic expression of SIRT1 in Hep1-6 cells activated a luciferase reporter construct, 832-529-luc, that covers both the fragments $803-662 \mathrm{bp}$ and $716-543 \mathrm{bp}$ (Figure 5F). Using reporter constructs containing a serial deletion, we identified a 93-bp fragment, 622-529 bp, which showed the highest level of Rictor promoter induction by SIRT1 (Figure 5F). This fragment is critical for Rictor induction, as its deletion markedly reduced the basal level of the reporter activity and completely blocked the promoter induction by SIRT1 (Figure 5F). Cotransfection of reporter 622-529 bp and SIRT1 plasmids increased luciferase activity of 622-529 bp, while expression of reporter 622-529 bp with either of the 2 SIRT1 knockdown siRNA oligos reduced the luciferase activity in Hepa1-6 cells (Figure 5G).

In the fragment of $622-529 \mathrm{bp}$, we identified 3 predicted nuclear respiratory factor 1-binding (NRF1-binding) sites (582-585 bp, 589-592 bp, and 618-621 bp) (Figure 5F). Our earlier data indicated SIRT1 interacts with NRF1 and positively regulates SIRT6 expression (40). Therefore, we suspected that NRF1 might be involved in the regulation of Rictor by SIRT1. In a reciprocal immunoprecipitation experiment, we demonstrated that SIRT1 and NRF1 interacted with each other (Figure 5H). Our further experiments found that, like SIRT1, NRF1 also interacts with the fragment 716-543 bp (Figure 5I). shRNA knockdown of NRF1 dramatically decreased the endogenous Rictor level (Figure 5J). At the same time, in luciferase activity assay, shRNA-mediated knockdown of NRF1 (Figure 5, J and K) not only reduced the basal level of the Rictor promoter reporter activity, it also blocked induction of Rictor by SIRT1 (Figure 5K). These data uncover an essential role for SIRT1 and NRF1 in maintaining Rictor expression.

It was previously shown that SIRT1 overexpression in transgenic mice causes a moderate increase of Nrf1 mRNA level (41). Thus, it is conceivable that SIRT1 deficiency could also reduce expression of NRF1, leading to decreased Rictor expression. To investigate this, we checked the NRF1 protein level in SIRT1 mutant liver and did not detect an obvious change in NRF1 expression (Supplemental Figure 4A). In Hepa1-6 cells, acute knockdown of SIRT1 also did not have an obvious change in NRF1 expression (Supplemental Figure 4B). Our data also indicated that NFR1 overexpression did not overcome the effect of SIRT1 deficiency on Rictor expression (Supplemental Figure 4C). Finally, we also overexpressed SIRT1 and found it did not affect NRF1 expression (Supplemental Figure 4D). This is consistent with the observation that SIRT1 and NRF1 interact with each other on the Rictor promoter that is required to maintain Rictor expression and rules out the possibility that the reduced Rictor expression is due to decreased NRF1 in SIRT1 mutant liver. 
Rictor negatively regulates hepatic glucose production. The data we obtained so far suggest that Rictor may mediate SIRT1 function in regulating hepatic glucose production. To investigate this further, we first performed shRNA-mediated acute knockdown of Rictor in wild-type primary hepatocytes. Two shRNAs against different sequences of Rictor significantly increased glucose production (Figure 6A). In the primary hepatocytes with Rictor knockdown, there was about a 2 -fold increase of glucose production in the presence of insulin (Figure 6A) compared with that in control knockdown cells.

We then knocked down Rictor in vivo by injecting the Rictor shRNA lentivirus into 3-month-old wild-type male mice through the tail vein. The mice were injected with control virus (shLuc) or Rictor shRNA virus (shRictor) twice, with 7 days between injections. One week after the second injection, PTT assay was carried out. Forty-five minutes after administration of pyruvate, the mice with shRictor produced significantly more glucose than the mice with shLuc. The increased level of glucose lasted until $100 \mathrm{~min}$ utes after pyruvate injection (Figure 6B). Glucose tolerance was also tested with this group of mice (Figure $6 \mathrm{C}$ ). The wild-type mice carrying shRictor displayed significant GTT intolerance during the 2-hour testing period. However, ITT did not reveal difference between the 2 groups of mice (data not shown). Finally, levels of Rictor and PAKT-S473 were assessed with the liver tissues from these mice (Figure 6, D and E). Lentivirus-based shRNA was able to significantly decrease Rictor level in the livers. The reduction of Rictor was accompanied with diminished pAKT-S473, both in insulin-stimulating situation (Figure 6D) and a normal fed condition (Figure 6E). We further showed knockdown of Rictor in the liver reduces Ser253 phosphorylation of FOXO1, which results in the activation of FOXO1 activity, illustrated by increased levels of G6pase, Pepck, and p21 at both protein and mRNA levels (Figure $6, F$ and $G$ ).

Next, we investigated whether adding back Rictor could restore FOXO1 inhibition. To perform this study, we delivered Rictor by lentivirus injection through the tail vein. The data showed that overexpression of Rictor inhibited the expression of FOXO1 downstream genes at both mRNA level and protein level (Figure 6, H and I). It also significantly improved GTT (Figure 6J) and increased AKT-S473 phosphorylation (Figure 6H). Because pAKT-S473 plays an essential role in regulating glucose production (20), our data suggest that the increased AKT activity may mediate the inhibition effect of Rictor in gluconeogenesis, although other mechanisms could not be ruled out.

Insulin resistance in Sirt $1^{\text {LKO }}$ mice is associated with increased intracellular ROS that can be reversed by antioxidants. We showed earlier that impaired hepatic function of SIRT1 causes increased hepatic glucose production and hyperglycemia, which eventually results in insulin resistance when animals get older. It has been shown that chronic overdosed glucose in the body may eventually cause glucose toxicity that is reflected mainly as increased ROS level (3841). To investigate this case, we measured the $\mathrm{H}_{2} \mathrm{O}_{2}$ level in young ( 2 months of age) and old (18 months of age) Sirt $1^{L K O}$ and control mice. Our analysis detected increased $\mathrm{H}_{2} \mathrm{O}_{2}$ only in brown adipose tissue (BAT) of young Sirt ${ }^{L K O}$ mice (Supplemental Figure 5A). In contrast, in old mutant mice, increased $\mathrm{H}_{2} \mathrm{O}_{2}$ had spread to more tissues, including white adipose tissue, skeletal muscle, and spleen, with the highest level in BAT (Figure 7A). Since most of these tissues are insulin-responsive tissues, next we examined members in the insulin signaling pathway. Our Western blot analysis revealed no obvious consistent changes in IRS-1, IRS-2, insulin receptor $\beta$ and $\alpha$, or PAKT-T308 in the tissues investigated (data not shown). However, it detected a marked reduction of AKT-S473 phosphorylation in these tissues (Figure 7B), while the total SIRT1 protein level remained the same (Figure 7C). Because reduced pAKT-S473 impairs insulin signaling, these data are in good correlation with the finding that $\operatorname{Sirt} 1^{L K O}$ mice developed insulin resistance.

We were initially surprised that these tissues had reduced PAKTS473, as we only deleted SIRT1 in the liver. Because these Sirt $1^{\text {LKO }}$ mice also exhibited elevated levels of $\mathrm{H}_{2} \mathrm{O}_{2}$, we suspected that the accumulation of intracellular ROS could serve as a cause for the reduced pAKT-S473. To prove this hypothesis, we treated cultured $293 \mathrm{HEK}$ cells with $\mathrm{H}_{2} \mathrm{O}_{2}$ and found that the treated cells lost the phosphorylation of AKT-S473 induced by insulin (Figure 7D), which is consistent with our finding that elevated $\mathrm{H}_{2} \mathrm{O}_{2}$ in the body reduced AKT-S473 phosphorylation. Since the level of PAKT-S473 is primarily controlled by the activity of mTORC2, we also followed the level of mTORC2 components. Our data detected no changes in the total amount of each component (mTOR, Rictor, and Sin 1 ) of the mTORC2 complex in the Sirt $1^{\text {LKO }}$ mice (Supplemental Figure 5B). Similar to what we found in tissues, $\mathrm{H}_{2} \mathrm{O}_{2}$-treated cells did not display changes in mTOR, Rictor, and Sin 1 protein levels (Figure 7D). Then, we asked whether it was possible that the complex formation of these proteins was affected. When coimmunoprecipitation was performed on these samples, $\mathrm{H}_{2} \mathrm{O}_{2}$-treated cells exhibited decreased binding of mTOR and Rictor to $\operatorname{Sin} 1$ (Figure 7E). To confirm this is the case in animal tissues, adipose tissues from control and Sirt $1^{L K O}$ mice were taken to perform an immunoprecipitation with antibody against $\operatorname{Sin} 1$. We detected a much lower association of Sin 1 with mTOR and Rictor in Sirt ${ }^{L K O}$ mouse adipose tissues (Figure 7F), suggesting that old Sirt $1^{L K O}$ mice became insulin intolerant due to increased $\mathrm{H}_{2} \mathrm{O}_{2}$ levels, which impaired the complex formation of mTORC2, and decreased pAKT-S473 levels in response to insulin treatment.

If the elevated level of $\mathrm{H}_{2} \mathrm{O}_{2}$ is a cause for insulin resistance, we reasoned that treatment with reagents that reduce oxidative stress or decrease intracellular ROS should at least partially reverse the insulin resistance in the Sirt $1^{L K O}$ mice. To test this, we treated

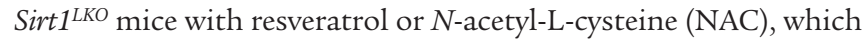
were reported to reduce intracellular $\operatorname{ROS}(42,43)$. Our data indicated that the treatment with either reagent significantly reduced the ROS level in multiple tissues of the Sirt $1^{\text {LKO }}$ mice (Figure 8A). We further showed that both reagents achieved similar significant improvement of insulin response compared with that of untreated mice, revealed by ITT (Figure 8, B and C), without an obvious effect on blood glucose levels (Supplemental Figure 5C) and hepatic AKT phosphorylation on S473 (Supplemental Figure 5D). Thus, these data indicate that ROS reduction is a major reason behind sensitization of Sirt $1^{\text {LKO }}$ mice to insulin.

\section{Discussion}

The importance of insulin and its downstream effecter AKT to glucose homeostasis has been studied extensively (42, 43). PDK1 and mTORC2 are both needed to fully activate AKT, which provides another layer of regulation downstream of the insulin-insulin receptor pathway (28). mTORC2 is composed of 2 unique components, Rictor and Sin 1 , and 2 common components, mTOR and mLST8, that are shared with mTORC1. Deletion of Rictor or Sin1 only impaired the phosphorylation of AKT at S473 upon insulin stimulation in MEFs $(39,44,45)$. Activation of AKT in hepatocytes 
results in the phosphorylation of the FOXO1 transcription factor, leading to its transportation to the cytoplasm and degradation (46). This results in the reduced expression of G6Pase and Pepck and diminished glucose production and elevated synthesis of glycogen in the liver. Conversely, the absence of AKT-S473 phosphorylation reduces the phosphorylation of FOXO1/FOXO3a, which is important for the maintenance of the transcriptional activity of FOXOs $(39,44)$. However, how mTORC2 expression and/or activity are regulated remains elusive. In this study, we showed that SIRT1-deficient liver has an impaired insulin response, primarily due to reduced phosphorylation of AKT-S473 in the presence of insulin. Our data demonstrated that SIRT1 controls the expression of Rictor, a component of the mTORC2, through interaction with NRF1 on the NRF1-binding sites of Rictor promoter. This relationship further illustrates the importance of a fine-tuning in the metabolic pathway and in maintaining insulin sensitivity.

In most cases, SIRT1 serves as a negative regulator of gene expression presumably due to its histone deacetylase activity. However, recent studies indicated that SIRT1 could activate gene expression through deacetylating or interacting with transcription factors in the promoter of downstream genes. It was shown that SIRT1 positively regulates PGC- $1 \alpha$-dependent gene expression through deacetylating PGC-1 $\alpha$, in terms of energy demand, such as under fasting and physical exercise conditions $(14,47)$. SIRT1 interacts with PPAR $\alpha$ and induces the expression of PPAR $\alpha$ target genes, including PGC-1 $\alpha$ (21). More recently, we found that SIRT1 forms a protein complex with NRF1 and FOXO3a on the SIRT6 promoter and maintains SIRT6 expression (40). Our data that SIRT1 and NRF1 form a protein complex on the NRF1 binding sites in the Rictor promoter is consistent with a positive role of SIRT1 on gene expression. We have also performed SIRT1 ChIP assay on G6Pase and Pepck promoter regions that are heavily guarded by histone acetylation modification and did not detect an enrichment of SIRT1 in these promoter regions (data not shown), ruling out the possibility that SIRT1 could directly regulate expression of these genes by modifying histone acetylation.

Abnormal regulation of hepatic glucose production is one of the causes for several human health problems, such as insulin resistance, type 2 diabetes, nonalcoholic fatty liver disease, and liver cirrhosis (48). In our study, we showed that all young Sirt $1^{\text {LKO }}$ animals initially suffered from hepatic glucose overproduction and hyperglycemia. They gradually developed insulin resistance as their age increased and eventually all exhibited whole-body insulin resistance, which is accompanied by increased levels of ROS in their insulin-response organs. Increased level of ROS is one of the major causes for insulin resistance (49-53). However, how ROS causes insulin resistance remains elusive. In the Sirt $1^{L K O}$ mice, we demonstrated that it is the hyperglycemia-induced ROS that results in the insulin resistance, because treating these mice with antioxidative reagents, resveratrol or NAC, significantly reversed this phenotype. Our study also uncovers a mechanism about how the hyperglycemia-induced ROS causes insulin resistance in many other tissues/organs, despite the fact that they are wild type for SIRT1. We found that ROS prevents mTORC2 protein complex formation by disassociating interactions among mTOR, Rictor, and Sin 1 . The failure of mTORC2 complex formation reduces phosphorylation of AKT-S473 and consequently promotes insulin resistance. This event is similar to our finding that SIRT1 deficiency in the liver decreases PAKT-S473 due to a reduced level of Rictor, a critical component of mTORC2, thereby underscoring an important role for SIRT1 in maintaining activity of mTORC2 complex and providing a mechanism how the hepatic insulin resistance caused by liver specific loss of SIRT1 eventually spread to other insulin-sensitive tissues/organs.

Our previous study revealed that about $30 \%$ of Sirt $1^{\text {LKO }}$ mice developed fatty liver starting at 2 months of age (22). Although liver steatosis is sometime correlated with overproduction of glucose (54), it is unlikely that fatty liver is a cause for hyperglycemia, as this lesion occurs in all Sirt ${ }^{L K O}$ mice at 2 months of age. In theory, fatty liver could serve as a cause for hepatic insulin resistance, but it is not always the case (reviewed in refs. 25, 54, 55). It is also shown that some animal models that suffer from liver steatosis had normal response to insulin, such as SIRT6 mutant mice (40). Thus, we believe that the effect of fatty liver on the development of insulin resistance in SIRT1 mutant mice is minor.

Previous studies also indicated that SIRT1 deacetylates FOXO1 at later stages of fasting and activates gluconeogenesis (19). Therefore, we have investigated the possibility that SIRT deficiency could affect acetylation of FOXO1. However, our analysis detected no obvious difference in FOXO1 acetylation between SIRT1 mutant and control mice at the normal feed condition, and the acetylated form of FOXO1 only slightly increased at 24 hours after fasting in the mutant liver compared with that in the control liver (Supplemental Figure 6). Since hyperglycemia is developed under the normal feed condition, which is accompanied by substantially reduced pFOXO1 S253, we believe the effect of SIRT1 on FOXO1 is primarily through phosphorylation, which targets FOXO1 to the cytoplasm for degradation. The effect of increased FOXO1 acetylation during fasting should be minor but is currently unclear. Upon short-term fasting (6 hours), SIRT1 is also found to repress glucose production through deacetylating CRTC2 (19). The impact of CRTC2 acetylation during short-term fasting on SIRT1 mutant mice is not clear and will be further investigated.

Of note, our finding that liver-specific disruption of SIRT1 increased gluconeogenesis is contradictory to previous studies of SIRT1 mutant mice, which did not report an obvious change in glucose production $(18,20,21)$. We have considered whether this discrepancy could be caused by a difference in genetic back-

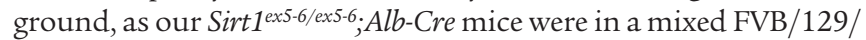
Black Swiss genetic background, and the other Sirt1 ${ }^{\text {ex4/ex4 }}$;Alb-Cre mice were in $\mathrm{C} 57 \mathrm{BL} / 6$ genetic background. To investigate this, we backcrossed our SIRT1 mutant mice 6 times into C57BL/ 6 genetic background (>98\%) and analyzed these mice when they were 6 weeks of age (Supplemental Figure 7). Our data indicate that the phenotypes of these mice, in terms of glucose production, gene expression, AKTS473 phosphorylation, and response to glucose challenge, are similar to SIRT1 mutant mice in mixed background. Based on these data, we do not believe that the genetic background is a major cause for difference between the mice in our study and the previously reported SIRT1 mutant mice. Because different mutant strains were used in the previous investigations (Sirt $1^{\text {ex4/ex4 }}$ )

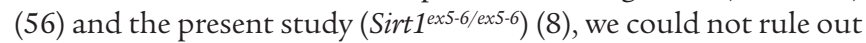
the possibility that the different mutant SIRT1 alleles could serve as a cause for the observed phenotypic difference, in addition to potential differences in animal housing and feeding conditions.

In summary, we showed that SIRT1 and NRF1 form a protein complex on the promoter of Rictor and positively regulate its expression. Hepatic-specific deletion of SIRT1 decreases the expression of Rictor, leading to reduced phosphorylation of AKTS473. This event maintains the nuclear localization of FOXO1 
and increases the expression of gluconeogenetic genes, G6pase and Pepck, leading to hepatic glucose overproduction and hyperglycemia. Insulin resistance in Sirt $1^{L K O}$ mice starts in the liver and gradually spreads to the whole body in older animal, due to chronic hyperglycemia-induced ROS in other organs/tissues. The increased ROS, in turn, disrupts mTORC2 complex formation and impairs Rictor-pAKT signaling in insulin-sensitive tissues, such as adipose tissues and muscle, leading to insulin resistance (Figure 8D). It has been reported that many human patients with type 1 diabetes who are treated with intensive insulin therapy from the onset of the disease eventually developed insulin resistance (57). Prolonged obesity conditions also frequently induce insulin resistance $(58,59)$. While the development of insulin resistance is complex and many unknown factors might be involved, it may be interesting to examine whether any patients might have increased ROS levels and impaired mTORC2 signaling, so that a personalized therapeutic approach against oxidative stress might be designed and tested. Our Sirt ${ }^{L K O}$ mice mimic the progressive insulin resistance caused by chronic hyperglycemia-induced ROS in human patients and could serve as an animal model for screening and testing preventive/therapeutic antioxidative drugs for overcoming human insulin resistance.

\section{Methods}

Animals. Male and female Sirt $1^{10 x 5-6} ;$ Alb-cre mice were intercrossed to generate Sirt $1^{L K O}$ mice and controls. These mice were either in a mixed genetic background of 129/FVB/Black Swiss or a C57BL/6 background (>98\%). Mice were maintained in 12-hour-light cycle environment. All the animal studies were carried out with 2-month-old males unless otherwise mentioned.

Glucose measurement. Mice were maintained under fed conditions with regular chow or fasted. At different ages, glucose was measured using a Contour glucose meter (Bayer) and Contour blood glucose test strips (Bayer).

$P T T$. Mice at 2 months of age were fasted overnight. After measuring the body weight and blood glucose level, $20 \%$ pyruvate in PBS was injected into each mouse i.p. at $100 \mu \mathrm{l} / 10 \mathrm{~g}$ body weight. The blood glucose level was monitored with a glucose meter at 15 -minute intervals during a 2-hour time course.

GTT. Mice at 2 months of age were fasted overnight. After measuring the body weight, $20 \%$ glucose was injected into each mouse i.p. at $100 \mu \mathrm{l} / 10 \mathrm{~g}$ body weight. The blood glucose level was measured with a glucose meter at 15 -minute intervals during a 2 -hour time course. In order to analyze insulin secretion during GTT, blood was collected 0, 15, and 120 minutes after glucose injection, and serum was prepared from these blood samples.

ITT. Mice at 2, 6, or 14 months of age were fasted for 4 hours. After measuring the body weight and glucose level, insulin (HumulinR, Lilly) was injected into each mouse i.p. $0.8 \mathrm{u} / \mathrm{kg}$ body weight. The blood glucose level was measured with a glucose meter at 15 -minute intervals during a 2-hour time course.

Hyperinsulinemic englycemic clamps. Eight pairs of 2-month-old mice underwent surgery and recovered for a week. After an overnight fast $(\sim 16$ hours), a 2-hour hyperinsulinemic euglycemic clamp was carried out in awake $\operatorname{Sirt} 1^{L K O}$ and wild-type mice. Detailed method are as follows. The clamp studies were performed in conscious, restrained mice. Four days before the clamp experiment, mice were anesthetized with $100 \mathrm{mg} / \mathrm{kg}$ ketamine and $10 \mathrm{mg} / \mathrm{kg}$ xylazine. A catheter was inserted into a lateral incision on the right side of the neck and advanced into the superior vena cava via the right internal jugular vein. The catheter was then sutured into place according to the protocol of MacLeod and Shapiro (60). Experiments were started at 1,000 hours, after a 4-hour fast. The basal rates of glucose turnover were measured by continuous infusion of $\left[3-{ }^{3} \mathrm{H}\right]$ glucose
$(0.05 \mu \mathrm{Ci} / \mathrm{min})$ for 120 minutes, which followed a bolus of $2 \mu \mathrm{Ci}$. Blood samples $(20 \mu \mathrm{l})$ were taken at 90 and 115 minutes of the basal period for the determination of plasma $\left[{ }^{3} \mathrm{H}\right]$ glucose concentration. A 120 -minutes hyperinsulinemic euglycemic clamp was started at 12:00 AM. Insulin was infused as a bolus of $18 \mathrm{mU} / \mathrm{kg}$ over a period of 3 minutes, followed by continuous insulin infusion at the rate of $2.5 \mathrm{mU} / \mathrm{kg} / \mathrm{min}$ (HumulinR; Eli Lilly) to raise the plasma insulin concentration to approximately $2 \mathrm{ng} / \mathrm{ml}$. During the clamp study, blood samples $(20 \mu \mathrm{l})$ were collected via a small nick in the tail vein at 15 -minute intervals for the immediate measurement of plasma glucose concentration, and $20 \%$ glucose was infused at variable rates to maintain plasma glucose at $140 \mathrm{mg} / \mathrm{dl}$. Insulin-stimulated whole-body glucose flux was estimated using a continuous infusion of high-pressure liquid chromatography-purified $\left[3-{ }^{3} \mathrm{H}\right]$ glucose $(0.1 \mu \mathrm{Ci} / \mathrm{min}$; NEN Life Science Products) throughout the clamps. To estimate insulin-stimulated glucose transport activity and metabolism in skeletal muscle, 2-deoxy-D- $\left[1-{ }^{14} \mathrm{C}\right]$ glucose (NEN Life Science Products) was administered as a bolus $(10 \mu \mathrm{Ci})$ at 45 minutes before the end of clamps. Blood samples $(20 \mu \mathrm{l})$ were taken at $80,85,90,100$, 110 , and 120 minutes after the start of clamps for the determination of plasma $\left[{ }^{3} \mathrm{H}\right]$ glucose, 2-deoxy-D-[ $\left[1-{ }^{14} \mathrm{C}\right]$ glucose, and ${ }^{3} \mathrm{H}_{2} \mathrm{O}$ concentrations. Additional blood samples $(10 \mu \mathrm{l})$ were collected before the start and at the end of clamp studies for measurements of plasma insulin concentration. All infusions were performed using microdialysis pumps (CMA/Microdialysis, Acton). At the end of the clamp period, animals were anesthetized with a ketamine-xylazine injection. Within 5 minutes, gastrocnemius muscle from hind limbs, epididymal and BAT, and liver were removed. Each tissue, once exposed, was dissected within 2 seconds, frozen immediately using liquid nitrogen-cooled aluminum blocks, and stored at $-70^{\circ} \mathrm{C}$ for later analysis.

Calculations. The determination of plasma [3-3H]glucose and 2-deoxyD-[1-14C]glucose concentrations and tissue 2-deoxy-D-[1-14C]glucose-6phosphate was performed as described previously (61). Basal endogenous glucose production was calculated as the ratio of the preclamp [3-3H] GIR $(\mathrm{dpm} / \mathrm{min})$ to the specific activity of the plasma glucose (mean of the values in the 90 - and 115 -minute basal preclamp period in $\mathrm{dpm} / \mu \mathrm{mol})$. Clamp whole-body glucose uptake was calculated as the ratio of the [3-3H]GIR $(\mathrm{dpm} / \mathrm{min})$ to the specific activity of plasma glucose $(\mathrm{dpm} / \mu \mathrm{mol})$ during the last 30 minutes of the clamp (mean of the 90- to 120-minute samples). Clamp endogenous glucose production was determined by subtracting the average GIR in the last 30 minutes of clamp from the whole-body glucose uptake. Muscle and white adipose tissue and BAT glucose uptake was calculated from the plasma 2-deoxy-D-[1-14C]glucose concentration profile (using plasma $14^{\circ} \mathrm{C}$ counts at $80-120$ minutes, the area under the curve was calculated by trapezoidal approximation) and tissue 2-deoxy-D[1-14C]glucose-6-phosphate content as described previously (61).

Insulin measurement. Serum was used for insulin measurements either with a Rat Insulin ELISA Kit (Crystal Chem Inc.) or by the RA method. Insulin was measured by the Metabolic Core Facility at National Institute of Diabetes and Digestive and Kidney Diseases.

Lentivirus injections. Male mice at 3 months of age were randomized into 2 groups. Through the tail vein, 1 group was injected with shLuciferase lentivirus; the other group was injected with shRictor, shFOXO1, or Rictor overexpressing lentivirus at 100 million virus/200 $\mu \mathrm{l} \mathrm{PBS/mouse.} \mathrm{All}$ the lentivirus vectors were purchased from OpenBio System as sets. After verifying every single vector's efficiency, the 2 most effective vectors were chosen to produce lentivirus. The injections were carried out twice, with 1 week between each other.

Isolation of primary hepatocyte and glucose production assay. Mouse hepatocytes were isolated via in situ collagenase perfusion and differential centrifugation on a Percoll gradient (Roche) as previously described (62). 
After seeding primary hepatocytes onto 24-well plates coated with collagen (Sigma-Aldrich) for 24 hours, cells were washed in PBS to remove glucose and were incubated in glucose production buffer (cAMP, dexamethasone, $20 \mathrm{mM}$ sodium lactate, and $2 \mathrm{mM}$ sodium pyruvate in PBS without $\mathrm{Mg}^{2+}$ and $\left.\mathrm{Ca}^{2+}[\mathrm{DPBS}]\right)$. When insulin treatment was applied, $100 \mathrm{nM}$ insulin was added to the culture 30 minutes before the samples were collected. Glucose concentrations were analyzed 6 hours later with the Glucose Assay Kit (G3293, Sigma-Aldrich), following the manufacturer's protocol, and normalized for protein concentration. When the lentivirus with shRNA against Rictor was used, the virus was incubated with hepatocytes for 3 days before the glucose production assay was carried out.

Western blot and immunoprecipitation. Western blot analysis was carried out with Licor, using antibodies against SIRT1 (Upstate); Rictor (Bethyl or Cell Signaling Technology); FOXO1, FOXO1S256, AKT, AKTS473, AKTT308, Pten, GSK $3 \alpha / \beta$, and $\mathrm{p} 70^{\mathrm{S} 6 \mathrm{~K}}$ (Cell Signaling Technology); acetylated FOXO1 and G6pase (Santa Cruz Biotechnology Inc.); NRF1 and PEPCK (Abcam); $\beta$-actin (Sigma-Aldrich); and Sin 1 (gift from Bin Su, Yale University, New Haven, Connecticut, USA). Immunoprecipitation was carried out as described previously (63). When liver tissue was collected from insulintreated mice, the mice were fasted for 6 hours, and then insulin was injected i.p. at $0.8 \mathrm{u} / \mathrm{kg}$ body weight. Livers were collected 30 minutes later.

qRT-PCR. Total RNA was isolated with STAT-60 (Tel-Test Inc.) from islets or Min 6 cell. cDNA was synthesized with Cells-to-cDNA II (Ambion Inc.). Quantitative RT-PCR was performed using a SYBR Green PCR Master Mix (Applied Biosystems) and 7500 Real-Time PCR (Applied Biosystems). Primers used for qRT-PCR are shown in Supplemental Table 1.

Cloning of promoters. To construct a murine Rictor promoter, a fragment from 852-bp to 529-bp upstream of ATG was cloned into PGL3B at the Mlu1-Xho1 site by PCR. After confirmation by sequencing, serial restriction enzyme deletion constructs were generated to nail down the response element to SIRT1.

Luciferase activity assay. PGL3B vectors containing different promoter sequences were transfected into Hepa1-6 or HepG2 cells. After a 24hours incubation, the luciferase activity was assessed with the DualLuciferase Reporter Assay Kit (Promega). The siRNA against murine NRF1 and SIRT1 were purchased from Dharmacon. Primers used for ChIP assay are shown in Supplemental Table 1.

ChIP assay on mRictor promoter. Hepa 1-6 cells were cross-linked with 1\% formalin for 15 minutes, and ChIP was performed with either SIRT1 or NRF1 (Abcam) antibody as described previously (63).

$\mathrm{H}_{2} \mathrm{O}_{2}$ measurement from tissues. The tissue $\mathrm{H}_{2} \mathrm{O}_{2}$ level was assessed by the Amplex Red Hydrogen Peroxide/Peroxidase Assay Kit (catalog no. A22188, Invitrogen), following the manufacture's protocol.
Transfection. All the transfections were carried out with Lipofectamine 2000 (Invitrogen). pUSE-Sirt1 is from Upstate. pCruz-SIRT1 and pCruz-Sirt1(HY) were described previously (7). shRNA against mouse SIRT1(pBSU6-Sirt1) was constructed as described previously (8). An shRictor lentivirus system was purchased from Open Biosystem. FOXO1 is from Origene.

IHC and immunofluorescence. Liver tissues were fixed with $10 \%$ formalin and embedded with paraffin. Five- $\mu \mathrm{m}$ sections were cut with a microtome MICROM (HM 340E). Primary hepatocytes grown on chamber slides were treated with or without $100 \mathrm{nM}$ insulin for 30 minutes and then fixed with $4 \%$ paraformaldehyde. Afterward, the sections or slides were stained with IHC or immunofluorescence.

Resveratrol and NAC treatment. The old wild-type or Sirt1 ${ }^{L K O}$ male mice were grouped together according to their age. ITT was performed to obtain their insulin response profile before the treatment started. The mice were then treated with either resveratrol $(0.8 \mu \mathrm{g} / \mathrm{ml}$ in drinking water) or NAC ( $1 \mathrm{~g} / \mathrm{l}$ in drinking water) for 9 weeks. Then ITT was performed on each group again to check their response to insulin. The ITT data are presented as the percentage over the base line (glucose level at each time point/starting glucose level).

Statistics. Student's $t$ test was used to compare differences between samples analyzed. $P$ values of less than 0.05 are considered as statistically significant. Data represent average \pm SEM.

Study approval. The experiments were approved by the Animal Care and Use Committee of the National Institute of Diabetes and Digestive and Kidney Diseases.

\section{Acknowledgments}

We gratefully acknowledge William Jou and Tatyana Chanturiya for technical assistance with clamps and serum analysis and members of the Deng laboratory for helpful discussion. This work was supported by the Intramural Research Program of the National Institute of Diabetes, and Digestive and Kidney Diseases, NIH, USA.

Received for publication December 27, 2010, and accepted in revised form August 16, 2011.

Address correspondence to: Chu-Xia Deng, Genetics of Development and Disease Branch, NIH, 10/9N105, 10 Center Drive, Bethesda, Maryland 20892, USA. Phone: 301.402.7225; Fax: 301.480.1135; E-mail: chuxiad@bdg10.niddk.nih.gov.
1. Saunders LR, Verdin E. Sirtuins: critical regulators at the crossroads between cancer and aging. Oncogene. 2007;26(37):5489-5504.

2. Lavu S, Boss O, Elliott PJ, Lambert PD. Sirtuins-novel therapeutic targets to treat age-associated diseases. Nat Rev Drug Discov. 2008;7(10):841-853.

3. Finkel T, Deng CX, Mostoslavsky R. Recent progress in the biology and physiology of sirtuins. Nature. 2009;460(7255):587-591.

4. Guarente L. Sirtuins in aging and disease. Cold Spring Harb Symp Quant Biol. 2007;72:483-488.

5. Vaquero A, Scher M, Erdjument-Bromage H, Tempst P, Serrano L, Reinberg D. SIRT1 regulates the histone methyl-transferase SUV39H1 during heterochromatin formation. Nature. 2007;450(7168):440-444.

6. Deng CX. SIRT1, is it a tumor promoter or tumor suppressor? Int J Biol Sci. 2009;5(2):147-152.

7. Wang RH, et al. Interplay among BRCA1, SIRT1, and Survivin during BRCA1-associated tumorigenesis. Mol Cell. 2008;32(1):11-20.

8. Wang RH, et al. Impaired DNA damage response, genome instability, and tumorigenesis in SIRT1 mutant mice. Cancer Cell. 2008;14(4):312-323.

9. Kim HS, et al. SIRT3 is a mitochondria-localized tumor suppressor required for maintenance of mitochondrial integrity and metabolism during stress. Cancer Cell. 2010;17(1):41-52.

10. Herranz D, Serrano M. SIRT1: recent lessons from mouse models. Nat Rev Cancer. 2010;10(12):819-823.

11. Lappalainen Z. Sirtuins: a family of proteins with implications for human performance and exercise physiology. Res Sports Med. 2011;19(1):53-65.

12. Nemoto S, Fergusson MM, Finkel T. Nutrient availability regulates SIRT1 through a forkhead-dependent pathway. Science. 2004;306(5704):2105-2108.

13. Motta MC, et al. Mammalian SIRT1 represses forkhead transcription factors. Cell. 2004;116(4):551-563.

14. Rodgers JT, Lerin C, Haas W, Gygi SP, Spiegelman BM, Puigserver P. Nutrient control of glucose homeostasis through a complex of PGC-1alpha and SIRT1. Nature. 2005;434(7029):113-118.

15. Rodgers JT, Lerin C, Gerhart-Hines Z, Puigserver P. Metabolic adaptations through the PGC- 1 alpha and SIRT1 pathways. FEBS Lett. 2008;582(1):46-53.

16. Erion DM, et al. SirT1 knockdown in liver decreases basal hepatic glucose production and increases hepatic insulin responsiveness in diabetic rats. Proc Natl Acad Sci U S A. 2009;106(27):11288-11293.

17. Banks AS, et al. SirT1 gain of function increases energy efficiency and prevents diabetes in mice. Cell Metab. 2008;8(4):333-341.

18. Wei D, Tao R, Zhang Y, White MF, Dong XC. Feedback regulation of hepatic gluconeogenesis through modulation of SHP/NrOb2 gene expression by Sirt 1 and FoxO1. Am J Physiol Endocrinol Metab. 2011;300(2):E312-E320.

19. Liu Y, et al. A fasting inducible switch modulates gluconeogenesis via activator/coactivator exchange. Nature. 2008;456(7219):269-273.

20. Chen D, et al. Tissue-specific regulation of SIRT1 by calorie restriction. Genes Dev. 2008;22(13):1753-1757.

21. Purushotham A, Schug TT, Xu Q, Surapureddi S, Guo X, Li X. Hepatocyte-specific deletion of SIRT1 alters fatty acid metabolism and results in hepatic steatosis and inflammation. Cell Metab. 
2009;9(4):327-338

22. Wang R-H, Li C, Deng C-X. Liver steatosis and increased ChREBP expression in mice carrying a liver specific SIRT1 null mutation under a normal feeding condition. Int J Biol Sci. 2010;6(7):682-690.

23. Nakae J, Park BC, Accili D. Insulin stimulates phosphorylation of the forkhead transcription factor FKHR on serine 253 through a Wortmannin-sensitive pathway. J Biol Chem. 1999; 274(23):15982-15985

24. Gross DN, van den Heuvel AP, Birnbaum MJ. The role of FoxO in the regulation of metabolism. Oncogene. 2008;27(16):2320-2336

25. Capeau J. Insulin resistance and steatosis in humans. Diabetes Metab. 2008;34(6 pt 2):649-657.

26. Alessi DR, et al. Characterization of a 3-phosphoinositide-dependent protein kinase which phosphorylates and activates protein kinase Balpha. Curr Biol. 1997;7(4):261-269.

27. Saltiel AR, Kahn CR. Insulin signalling and the regulation of glucose and lipid metabolism. Nature. 2001;414(6865):799-806.

28. Sarbassov DD, Guertin DA, Ali SM, Sabatini DM. Phosphorylation and regulation of Akt/ PKB by the rictor-mTOR complex. Science. 2005 307(5712):1098-1101.

29. Sabatini DM. mTOR and cancer: insights into a complex relationship. Nat Rev Cancer. 2006; 6(9):729-734.

30. O'Brien RM, Streeper RS, Ayala JE, Stadelmaier BT, Hornbuckle LA. Insulin-regulated gene expression. Biochem Soc Trans. 2001;29(pt 4):552-558.

31. O'Brien RM, Granner DK. Regulation of gene expression by insulin. Physiol Rev. 1996;76(4):1109-1161.

32. Agati JM, Yeagley D, Quinn PG. Assessment of the roles of mitogen-activated protein kinase, phosphatidylinositol 3-kinase, protein kinase $\mathrm{B}$, and protein kinase $C$ in insulin inhibition of cAMP-induced phosphoenolpyruvate carboxykinase gene transcription. J Biol Chem. 1998;273(30):18751-18759.

33. Sutherland C, O'Brien RM, Granner DK. Phosphatidylinositol 3-kinase, but not p70/p85 ribosomal S6 protein kinase, is required for the regulation of phosphoenolpyruvate carboxykinase (PEPCK) gene expression by insulin. Dissociation of signaling pathways for insulin and phorbol ester regulation of PEPCK gene expression. J Biol Chem. 1995;270(26):15501-15506

34. Schmoll D, et al. Regulation of glucose-6phosphatase gene expression by protein kinase Balpha and the forkhead transcription factor FKHR. Evidence for insulin response unit-dependent and -independent effects of insulin on promoter activity. J Biol Chem. 2000;275(46):36324-36333.

35. Huang H, Tindall DJ. Dynamic FoxO transcription factors. J Cell Sci. 2007;120(pt 15):2479-2487.
36. Foufelle F, Ferre P. New perspectives in the regulation of hepatic glycolytic and lipogenic genes by insulin and glucose: a role for the transcription factor sterol regulatory element binding protein1c. Biochem J. 2002;366(pt 2):377-391.

37. Vander Kooi BT, et al. The glucose-6-phosphatase catalytic subunit gene promoter contains both positive and negative glucocorticoid response elements. Mol Endocrinol. 2005;19(12):3001-3022.

38. Barthel A, et al. Differential regulation of endogenous glucose-6-phosphatase and phosphoenolpyruvate carboxykinase gene expression by the forkhead transcription factor FKHR in H4IIEhepatoma cells. Biochem Biophys Res Commun. 2001;285(4):897-902.

39. Jacinto E, et al. SIN1/MIP1 maintains rictormTOR complex integrity and regulates Akt phosphorylation and substrate specificity. Cell. 2006; 127(1):125-137.

40. Kim H-S, et al. Hepatic-specific disruption of SIRT6 in mice results in fatty liver formation due to enhanced glycolysis and triglyceride synthesis. Cell metabolism. 2010;12(3):224-236.

41. Pfluger PT, Herranz D, Velasco-Miguel S, Serrano M, Tschop MH. Sirt1 protects against high-fat dietinduced metabolic damage. Proc Natl Acad Sci US A. 2008;105(28):9793-9798.

42. Michael MD, et al. Loss of insulin signaling in hepatocytes leads to severe insulin resistance and progressive hepatic dysfunction. Mol Cell. 2000 6(1):87-97.

43. Taniguchi CM, Emanuelli B, Kahn CR. Critical nodes in signalling pathways: insights into insulin action. Nat Rev Mol Cell Biol. 2006;7(2):85-96.

44. Yang Q, Inoki K, Ikenoue T, Guan KL. Identification of Sin 1 as an essential TORC 2 component required for complex formation and kinase activity. Genes Dev. 2006;20(20):2820-2832.

45. Guertin DA, et al. Ablation in mice of the mTORC components raptor, rictor, or mLST8 reveals that mTORC2 is required for signaling to AktFOXO and PKCalpha, but not S6K1. Dev Cell. 2006;11(6):859-871.

46. Gross DN, Wan M, Birnbaum MJ. The role of FOXO in the regulation of metabolism. Curr Diab Rep. 2009;9(3):208-214

47. Gerhart-Hines $Z$, et al. Metabolic control of muscle mitochondrial function and fatty acid oxidation through SIRT1/PGC-1alpha. EMBO J. 2007;26(7):1913-1923

48. Marchesini G, et al. Nonalcoholic fatty liver disease: a feature of the metabolic syndrome. Diabetes. 2001;50(8):1844-1850.

49. Brownlee M. Biochemistry and molecular cell biology of diabetic complications. Nature. 2001; 414(6865):813-820.
50. Veal EA, Day AM, Morgan BA. Hydrogen peroxide sensing and signaling. Mol Cell. 2007;26(1):1-14.

51. Newsholme P, et al. Diabetes associated cell stress and dysfunction: role of mitochondrial and nonmitochondrial ROS production and activity. J Physiol. 2007;583(pt 1):9-24.

52. Kim JA, Wei Y, Sowers JR. Role of mitochondrial dysfunction in insulin resistance. Circ Res. 2008;102(4):401-414.

53. Lee HY, et al. Targeted expression of catalase to mitochondria prevents age-associated reductions in mitochondrial function and insulin resistance. Cell Metab. 2010;12(6):668-674.

54. Bugianesi E, Moscatiello S, Ciaravella MF, Marchesini G. Insulin resistance in nonalcoholic fatty liver disease. Curr Pharm Des. 2010;16(17):1941-1951.

55. Bugianesi E, Zannoni C, Vanni E, Marzocchi R, Marchesini G. Non-alcoholic fatty liver and insulin resistance: a cause-effect relationship? Dig Liver Dis. 2004;36(3):165-173

56. Cheng HL, et al. Developmental defects and p53 hyperacetylation in Sir2 homolog (SIRT1)deficient mice. Proc Natl Acad Sci U S A. 2003; 100(19):10794-10799.

57. Araszkiewicz A, Uruska A, Zozulinska-Ziolkiewicz D, Pilacinski S, Wierusz-Wysocka B. Factors related to insulin resistance in type 1 diabetic patients treated with intensive insulin therapy from the onset of the disease. Diabetes Res Clin Pract. 2010;90(2):e23-e24

58. Sakurai T, et al. Age-associated increase in abdominal obesity and insulin resistance, and usefulness of AHA/NHLBI definition of metabolic syndrome for predicting cardiovascular disease in Japanese elderly with type 2 diabetes mellitus. Gerontology. 2010;56(2):141-149.

59. Fleischman A, Kron M, Systrom DM, Hrovat M, Grinspoon SK. Mitochondrial function and insulin resistance in overweight and normal-weight children. JClin Endocrinol Metab. 2009;94(12):4923-4930.

60. MacLeod JN, Shapiro BH. Repetitive blood sampling in unrestrained and unstressed mice using a chronic indwelling right atrial catheterization apparatus. Lab Anim Sci. 1988;38(5):603-608.

61. Youn JH, Kim JK, Buchanan TA. Time courses of changes in hepatic and skeletal muscle insulin action and GLUT4 protein in skeletal muscle after STZ injection. Diabetes. 1994;43(4):564-571.

62. Jeong WI, et al. Paracrine activation of hepatic CB1 receptors by stellate cell-derived endocannabinoids mediates alcoholic fatty liver. Cell Metab. 2008;7(3):227-235.

63. Wang RH, Yu H, Deng CX. A requirement for breast-cancer-associated gene 1 (BRCA1) in the spindle checkpoint. Proc Natl Acad Sci U S A. 2004;101(49):17108-17113. 